\title{
Nonsymmetric gravity theories: Inconsistencies and a cure
}

\author{
T. Damour \\ Institut des Hautes Etudes Scientifiques, 91440 Bures sur Yvette, France \\ and Département d'Astrophysique Relativiste et de Cosmologie, Centre National de la Recherche Scientifique, \\ Observatoire de Paris, 92195 Meudon, France \\ S. Deser \\ Physics Department, Brandeis University, Waltham, Massachusetts 02254 \\ J. McCarthy \\ Department of Physics and Mathematical Physics, University of Adelaide, \\ GPO Box 498, Adelaide, South Australia 5001, Australia
}

(Received 26 June 1992)

\begin{abstract}
Motivated by the apparent dependence of string $\sigma$ models on the sum of spacetime metric and antisymmetric tensor fields, we reconsider gravity theories constructed from a nonsymmetric metric. We first show, by expanding in powers of the antisymmetric field, that all such "geometrical" theories homogeneous in second derivatives violate standard physical requirements: ghost freedom, absence of algebraic inconsistencies, or continuity of degree-of-freedom content. This no-go result applies in particular to the old unified theory of Einstein and its recent avatars. However, we find that the addition of nonderivative, "cosmological" terms formally restores consistency by giving a mass to the antisymmetric tensor field, thereby transmuting it into a fifth-force-like massive vector but with novel possible matter couplings. The resulting macroscopic models also exhibit "van der Waals"-type gravitational effects, and may provide useful phenomenological foils to general relativity.
\end{abstract}

PACS number(s): 04.50.th

\section{INTRODUCTION}

It is a remarkable historical coincidence that modern string theory can be interpreted as reviving an ancient attempt at geometric unification of forces. Specifically, we note that (ignoring the dilaton for simplicity) the string $\sigma$ model action in conformal gauge is just

$$
I=\int d^{2} z\left[G_{\mu \nu}(X)+B_{\mu \nu}(X)\right] \partial_{z} X^{\mu} \partial_{\bar{z}} X^{\nu}
$$

in terms of local complex world-sheet coordinates $z, \bar{z}$. Here $X^{\mu}$ is the embedding coordinate of the string in a $D$-dimensional spacetime endowed with the (symmetric) metric $G_{\mu \nu}$ and the antisymmetric tensor field $B_{\mu \nu}$. The action (1.1) thus depends only on the "unified" combination

$$
g_{\mu \nu} \equiv G_{\mu \nu}+B_{\mu \nu},
$$

and we are led to consider field theories of gravity constructed geometrically from the nonsymmetric metric $g_{\mu \nu}$. One such model was first discussed in 1925 by Einstein [1] in an attempt to unify gravity and electromagnetism ( $B_{\mu \nu}$ was to be related to the Maxwell field strength). Further treatment by Einstein and his collaborators [2], Schrödinger [3], and others [4-6] led to considerable elaboration of its mathematical structure. More recently, Moffat [7] has proposed that $B_{\mu \nu}$ be interpreted as a new field coupled to macroscopic currents. Following recent usage, we shall refer to this specific theory as NGT ("nonsymmetric gravity theory"), but we shall also analyze the whole class of geometric nonsymmetric theories.

A priori, nonsymmetric geometrization suffers from a number of weaknesses. Not least is the absence of a natural way to unify the two initial building blocks $G_{\mu \nu}$ and $B_{\mu \nu}$ into a single entity. This objection was originally raised by Weyl [8] and Pauli [9] in connection with Einstein's attempts; it states that because $g_{\mu \nu}$ is a reducible representation of diffeomorphisms, there is no real meaning in saying that a theory is expressed "solely in terms of $g_{\mu \nu}$." Indeed, any theory involving $G_{\mu \nu}$ and $B_{\mu \nu}$ can always be written in terms of $g_{\mu \nu}$, since these variables can of course simply be expressed as the symmetric and antisymmetric parts of $g_{\mu \nu}$. One might hope that this objection could be removed by requiring the theory to be "geometrically constructed," a notion still to be defined, but we shall see that even this constraint turns out not to be sufficient. A more concrete problem is that the string $\sigma$ model, and hence the perfectly consistent but nongeometric effective field theory it generates, is invariant under the local gauge transformation

$$
\delta_{0} B_{\mu \nu}=\partial_{\mu} \epsilon_{\nu}-\partial_{\nu} \epsilon_{\mu},
$$

in addition to the usual diffeomorphism invariance. Hence all dependence on $B_{\mu \nu}$ must be through the invariant field strength tensor

$$
H_{\lambda \mu \nu} \equiv \partial_{\lambda} B_{\mu \nu}+\partial_{\mu} B_{\nu \lambda}+\partial_{\nu} B_{\lambda \mu}
$$

and indeed the effective string expansion (which is in powers of derivatives, thereby maintaining consistency) 
begins as the sum of Einstein plus $H_{\lambda \mu \nu}^{2}$ terms. This puts the effective theory at odds with generic geometric models constructed from $g_{\mu \nu}$ with the number of derivatives fixed at two, unless such models can maintain the above invariance - which will turn out to be crucial to their consistency. Nevertheless, despite these a priori difficulties of the geometric approach, it merits reexamination not only in view of the string motivation, but because we will provide a simple (geometric) extension of these ideas that restores consistency.

In this paper, we will analyze the generic geometric models, by expanding them in powers of $B_{\mu \nu}$ about a classical symmetric background, and consider the merits of the resulting "gravity + matter" theories in terms of standard physical criteria: absence of negative-energy excitations ("ghosts"), of algebraic inconsistencies, or discontinuities in the degree-of-freedom content. Our results are negative for the "geometric" two-derivative theories. In brief, our argument is that consistency requires the $B$ expansion to begin with (quadratic) kinetic terms of $H_{\mu \nu \lambda}^{2}$ form [10], whereas geometric actions homogeneous in two derivatives produce extensions which necessarily include dependence on powers of undifferentiated $B_{\mu \nu}$. These higher-power terms generically violate the gauge invariance of the leading kinetic term, with the usual consequences that there are either ghosts, algebraic inconsistencies, or unacceptable constraints on "independent" degrees of freedom. For instance, as we already pointed out in [11], NGT suffers from curvature-coupled ghosts. (Of course the fact that the one consistent, $R+H^{2}$, two-derivative model can be formally written as an infinite series of "geometric" terms is not to be regarded as a counterexample.) We will then show that restoration of consistency can nevertheless be achieved within the framework of geometric theories by adding "cosmological," nonderivative terms which render the $B$ field massive, thereby sidestepping the problems of gauge inconsistencies. Such extensions will be seen to provide viable alternative gravity models to be confronted with observation.

In Sec. II, we introduce appropriate definitions of geometric theories constructed from nonsymmetric metrics, and the associated expansion in $B_{\mu \nu}$. [We also settle there and in Appendix B certain questions raised in the literature concerning equivalence of first- (Palatini) and second-order formulations.] In Sec. III, we enumerate the generic difficulties faced by these models and prove that they cannot be overcome by deformation of the Abelian gauge invariance (1.3). Section IV shows in detail how the two main previously proposed models fail. In Sec. V, we introduce a new class of consistent geometric gravity models endowing $B_{\mu \nu}$ with a finite range, couple it to macroscopic sources and discuss some possible observational consequences. All our considerations are intended to be at the purely classical, low-energy level.

In view of the sometimes confusing statements in the literature, it may be useful to spell out some of our basic assumptions. The first of these is that it is meaningful (if not outright mandatory), particularly at the macroscopic level, to expand the theories in terms of their two independent coupling constants: the usual gravitational one and a corresponding one for the $B$ field. The theory must obviously reduce to ordinary Einstein gravity when the latter is neglected. Secondly, we make the physical requirement that, when so expanded, the theory have no ghost-negative energy - modes. For, even at the classical level, presence of such modes in the excitation spectrum simply means, if they fail to decouple, that the universe is liable to be unstable to their radiation. Indeed, we will see concretely that, in NGT, such an instability is unavoidable irrespective of any choice of initial conditions.

\section{GEOMETRIC THEORIES}

In the standard tradition, a geometric theory is defined by an action constructed from generally covariant objects built with the metric tensor $g_{\mu \nu}$ and the affine connection $\Gamma_{\mu \nu}^{\lambda}$ on the spacetime manifold. The affinity may either be an independent field (first order formulation) or a prescribed function of $g_{\mu \nu}$ (second order). These concepts can be largely carried over to models whose metric tensor and affinity are no longer symmetric. The Riemann curvature tensor is still

$$
R_{\nu \alpha \beta}^{\mu}=\partial_{\alpha} \Gamma_{\nu \beta}^{\mu}-\Gamma_{\nu \alpha}^{\sigma} \Gamma_{\sigma \beta}^{\mu}-[\alpha \leftarrow \rightarrow \beta],
$$

but there are now two possible (metric-independent) contractions,

$$
R_{\mu \nu}=R_{\mu \lambda \nu}^{\lambda}, \quad P_{\mu \nu}=R_{\lambda \mu \nu}^{\lambda} .
$$

Note that while $P_{\mu \nu}$ is antisymmetric, $R_{\mu \nu}$ has no symmetries. To construct a scalar curvature requires an inverse, $g^{\mu \nu}$, which we define by the usual convention

$$
g^{\mu \alpha} g_{\nu \alpha}=g^{\alpha \mu} g_{\alpha \nu}=\delta_{\nu}^{\mu}\left(\neq g^{\mu \alpha} g_{\alpha \nu}\right) \text {. }
$$

The antisymmetric part of the connection is the torsion tensor

$$
T_{\mu \nu}^{\lambda}=\frac{1}{2} \Gamma_{[\mu \nu]}^{\lambda} \equiv \frac{1}{2}\left(\Gamma_{\mu \nu}^{\lambda}-\Gamma_{\nu \mu}^{\lambda}\right) .
$$

Throughout, we denote unnormalized antisymmetrization (symmetrization) by square (round) brackets.

When expanding geometric objects in powers of $B_{\mu \nu}$, we assume the symmetric part $G_{\mu \nu}$ to be invertible, and use it to move indices. Of course, the naive split (1.2) can always be composed with a local algebraic field redefinition, replacing (1.2) by

$$
g_{\mu \nu}=G_{\mu \nu}+B_{\mu \nu}+\alpha B_{\mu \alpha} B^{\alpha}{ }_{\nu}+\beta B^{\alpha \beta} B_{\alpha \beta} G_{\mu \nu}+O\left(B^{3}\right) \text {. }
$$

Note that the generic form (2.5) incorporates the freedom of defining the irreducible parts by decomposing a different "metric" field variable, say $(\sqrt{-g})^{n} g^{\mu \nu}$, so there is no loss of generality in this respect. For convenience, we have collected the relevant expansions in Appendix A.

A geometric theory in first-order form is one whose action depends on $g$ and $\Gamma$ through the curvatures defined above, as well as on invariants - built from torsionwhich dimensionally respect the second-derivative re- 
quirement. For concreteness, consider NGT which is the simplest generalization of general relativity in Palatini form,

$$
\mathcal{L}_{\mathrm{NGT}}^{(1)}(g, \Gamma)=\sqrt{-g} g^{\mu \nu} R_{\mu \nu}(\Gamma) .
$$

In $D$ dimensions, its field equations are

$$
\begin{aligned}
& (-g)^{-1 / 2} \partial_{\lambda}\left(\sqrt{-g} g^{\mu \nu}\right)+\Gamma_{\alpha \lambda}^{\mu} g^{\alpha \nu}+\Gamma_{\lambda \alpha}^{\nu} g^{\mu \alpha} \\
& \quad-\Gamma_{\alpha \lambda}^{\alpha} g^{\mu \nu}-2 \Gamma_{\lambda} g^{\mu \nu}+\frac{2}{D-1} \delta_{\lambda}^{\nu} \Gamma_{\alpha} g^{\mu \alpha}=0 \\
& R_{\mu \nu}(\Gamma)-\frac{1}{2} g_{\mu \nu} R=0
\end{aligned}
$$

where $\Gamma_{\mu} \equiv \frac{1}{2} \Gamma_{[\mu \lambda]}^{\lambda}$ and $R \equiv g^{\mu \nu} R_{\mu \nu}(\Gamma)$. For $D>2$, (2.7) is equivalent to

$$
\partial_{\lambda} g_{\mu \nu}-\widetilde{\Gamma}_{\mu \lambda}^{\alpha} g_{\alpha \nu}-\widetilde{\Gamma}_{\lambda \nu}^{\alpha} g_{\mu \alpha}=0,
$$

where

$$
\widetilde{\Gamma}_{\mu \nu}^{\lambda} \equiv \Gamma_{\mu \nu}^{\lambda}+\frac{2}{D-1} \delta_{\mu}^{\lambda} \Gamma_{\nu}
$$

is constrained by the condition

$$
\widetilde{\Gamma}_{[\mu \lambda]}^{\lambda}=0 \text {. }
$$

Combining (2.10) and (2.11) with (2.9) implies

$$
\partial_{\mu}\left(\sqrt{-g} g^{[\mu \nu]}\right)=0 \text {. }
$$

The superficial resemblance of (2.8) and (2.9) to the usual Einstein-Palatini system must be viewed with some caution. One obvious difference is the fact that now just $\widetilde{\Gamma}_{\mu \nu}^{\lambda}$, but not $\Gamma_{\mu}$, is determined by (2.9) in terms of $g_{\mu \nu}$. Instead, $\Gamma_{\mu}$ plays the role of a Lagrange multiplier imposing the constraint (2.12). To see this, note that

$$
R_{\mu \nu}(\Gamma)=R_{\mu \nu}(\widetilde{\Gamma})-\frac{2}{D-1} \partial_{[\mu} \Gamma_{\nu]}
$$

so the Lagrangian $\mathcal{L}_{\mathrm{NGT}}^{(1)}$ splits into

$$
\mathcal{L}_{\mathrm{NGT}}^{(1)}\left(g, \widetilde{\Gamma}, \Gamma_{\mu}\right)=\sqrt{-g} g^{\mu \nu}\left[R_{\mu \nu}(\widetilde{\Gamma})-\frac{2}{D-1} \partial_{[\mu} \Gamma_{\nu]}\right],
$$

then integrate by parts in the second term of (2.14). [In deriving the field equations from (2.14) one must take account of the constraint (2.11).] A second difference from symmetric theories is that neither the metric nor the affinity have their familiar symmetry properties, and thus the index ordering in (2.9) is nontrivial. As was emphasized by Schrödinger, (2.9) gives a well-defined expansion for $\widetilde{\Gamma}_{\mu \nu}^{\lambda}$ in powers of $B_{\mu \nu}$. [Actually it has been shown by Tonnelat [5] that (2.9) can be solved in closed form; however, the resulting expression is extremely complicated.] This is by no means the only ordering which does so since, for example, torsion terms may be added to (2.9) as follows

$$
\begin{aligned}
\partial_{\lambda} g_{\mu \nu}-\widetilde{\Gamma}_{\mu \lambda}^{\alpha} g_{\alpha \nu}-\widetilde{\Gamma}_{\lambda \nu}^{\alpha} g_{\mu \alpha} \\
\quad=a \widetilde{T}_{\mu \lambda}^{\alpha} g_{\alpha \nu}+b \widetilde{T}_{\lambda \nu}^{\alpha} g_{\mu \alpha}+c \widetilde{T}_{\mu \lambda}^{\alpha} g_{\nu \alpha}+d \widetilde{T}_{\lambda \nu}^{\alpha} g_{\alpha \mu},
\end{aligned}
$$

without altering either its tensorial character or its generic solvability. [Indeed the tensorial nature of (2.9) is clearest from the fact that its LHS differs from the usual covariant derivative of $g_{\mu \nu}$ by a torsion term.] There is, however, a line of choices in parameter space, namely $a+b+c+d+2=0$, for which (2.15) cannot be used to determine $\widetilde{\Gamma}_{\mu \nu}^{\lambda}$. We discuss this issue more fully in Appendix A.

Because torsion is available, the above EinsteinPalatini generalization may be further extended ${ }^{1}$ (while maintaining the second-derivative requirement) to

$$
\begin{aligned}
\mathcal{L}_{\text {general }}^{(1)}(g, \Gamma)=\sqrt{-g} g^{\mu \nu}[ & R_{\mu \nu}(\Gamma)+a_{1} P_{\mu \nu}(\Gamma)+a_{2} \partial_{[\mu} \Gamma_{\nu]}+b_{1} \nabla_{\lambda} T_{\mu \nu}^{\lambda}+b_{2} T_{\mu \alpha}^{\lambda} T_{\lambda \nu}^{\alpha}+b_{3} T_{\mu \nu}^{\lambda} \Gamma_{\lambda} \\
& \left.+c_{1} g^{\lambda \delta} g_{\alpha \beta} T_{\mu \lambda}^{\alpha} T_{\nu \delta}^{\beta}+c_{2} g^{\lambda \delta} g_{\alpha \beta} T_{\mu \nu}^{\alpha} T_{\lambda \delta}^{\beta}+c_{3} g^{\lambda \delta} g_{\alpha \beta} T_{\mu \delta}^{\alpha} T_{\nu \lambda}^{\beta}+d_{1} \Gamma_{\mu} \Gamma_{\nu}\right] .
\end{aligned}
$$

To understand the nature of the additional terms in light of the analysis above, let us again decompose $\Gamma_{\mu \nu}^{\lambda}$ as in (2.10), to rewrite this as

$$
\begin{aligned}
\mathcal{L}_{\text {general }}^{(1)}\left(g, \widetilde{\Gamma}, \Gamma_{\mu}\right)= & \sqrt{-g} g^{\mu \nu}\left[R_{\mu \nu}(\widetilde{\Gamma})+a_{1} \partial_{[\mu} \widetilde{\Gamma}_{\nu] \lambda}^{\lambda}+b_{1} \widetilde{\nabla}_{\lambda} \widetilde{T}_{\mu \nu}^{\lambda}+b_{2} \widetilde{T}_{\mu \alpha}^{\lambda} \widetilde{T}_{\lambda \nu}^{\alpha}\right. \\
& \left.\quad+c_{1} g^{\lambda \delta} g_{\alpha \beta} \widetilde{T}_{\mu \lambda}^{\alpha} \widetilde{T}_{\nu \delta}^{\beta}+c_{2} g^{\lambda \delta} g_{\alpha \beta} \widetilde{T}_{\mu \nu}^{\alpha} \widetilde{T}_{\lambda \delta}^{\beta}+c_{3} g^{\lambda \delta} g_{\alpha \beta} \widetilde{T}_{\mu \delta}^{\alpha} \widetilde{T}_{\nu \lambda}^{\beta}\right] \\
+ & \Gamma_{\lambda}\left[\left(\frac{2}{D-1}+\frac{2 D}{D-1} a_{1}-a_{2}+\frac{b_{1}}{D-1}\right) \partial_{\mu}\left(\sqrt{-g} g^{[\mu \lambda]}\right)+b_{3} \widetilde{T}_{\mu \nu}^{\lambda} \sqrt{-g} g^{\mu \nu}\right. \\
& \left.\quad+\frac{c_{2}}{D-1} \widetilde{T}_{\mu \nu}^{\beta} \sqrt{-g}\left(g_{\alpha \beta} g^{\lambda \alpha}-g_{\beta \alpha} g^{\alpha \lambda}\right) g^{\mu \nu}+\frac{c_{3}}{D-1} \widetilde{T}_{\mu \nu}^{\beta}\left(g^{\mu \lambda} g_{\beta \alpha} g^{\alpha \nu}+g^{\lambda \mu} g_{\alpha \beta} g^{\nu \alpha}\right)\right] \\
& +\left[\left(d_{1}-\frac{b_{2}}{D-1}+\frac{c_{2}}{(D-1)^{2}}+\frac{2}{D-1}\left(c_{1}+c_{3}\right)\right) \sqrt{-g} g^{\mu \nu}-\frac{c_{2}}{(D-1)^{2}} \sqrt{-g} g_{\alpha \beta} g^{\mu \alpha} g^{\beta \nu}\right] \Gamma_{\mu} \Gamma_{\nu} .
\end{aligned}
$$

\footnotetext{
${ }^{1}$ Historically, NGT was selected by Einstein because it possesses two (physically ill-motivated [9]) symmetries: transposition invariance $\left(g_{\mu \nu} \rightarrow g_{\nu \mu}, \widetilde{\Gamma}_{\mu \nu}^{\lambda} \rightarrow \widetilde{\Gamma}_{\nu \mu}^{\lambda}, \Gamma_{\mu} \rightarrow-\Gamma_{\mu}\right)$ and " $\lambda$-invariance" $\left(\Gamma_{\mu} \rightarrow \Gamma_{\mu}+\partial_{\mu} \lambda\right)$.
} 
The result is that $\Gamma_{\mu}$ still imposes a constraint in some sense - the analogy to gauge-fixing is unmistakable, though as we will see, misleading. (Certainly such a gauge-fixing interpretation is untenable unless $b_{3}=$ $c_{2}=c_{3}=0$, since otherwise it is a nonlinear "gauge" and Faddeev-Popov ghosts would be required. Accepting this constraint, the model-since it contains the multiplier $\Gamma_{\mu}$ both linearly and quadratically-resembles a choice parametrized between "Landau" and "Fermi" type gauges.) We will not need to use the full generality in (2.16) in the following; it just serves to show that nothing very dramatic occurs if we allow it, and that the generalizations (2.15) can be obtained from an action. The models in which all parameters but $a_{1}$ and $a_{2}$ are set to zero have been especially discussed in the literature. It is clear from (2.17) that if, further, the coefficient of the " $\Gamma_{\mu}$ constraint" is chosen to vanish [i.e., $\left.2+2 D a_{1}-(D-1) a_{2}=0\right]$, then we have an exceptional case, but otherwise these models are all equivalent [7] to NGT, Eq. (2.6), and stand or fall with it.

Those members of the general class (2.16) for which the affinity cannot be solved in terms of the metric are not viable geometric theories and we do not consider them any further. On the other hand, all other models can of course be expressed in second-order form, i.e., in terms of affinities which are explicit functions of the metric. We need therefore only deal with second-order formulations henceforth. For simplicity, we define $\Gamma(g)$ by what Einstein called the "+-" relation

$$
\partial_{\lambda} g_{\mu \nu}-\Gamma_{\mu \lambda}^{\alpha} g_{\alpha \nu}-\Gamma_{\lambda \nu}^{\alpha} g_{\mu \alpha}=0 \text {. }
$$

Second-order geometric theories are then defined by the requirement that the action depend on $g_{\mu \nu}$ (and its inverses) with all derivatives appearing in general covariants constructed from $\Gamma_{\mu \nu}^{\lambda}(g)$ defined through (2.18).

As for all theories involving torsion, there is of course a difference between the first-order theory (2.6) and the naive second-order theory defined by the same Lagrangian with $\Gamma_{\mu \nu}^{\lambda}=\Gamma_{\mu \nu}^{\lambda}(g)$ determined by (2.18) [see (2.22) below]. From the discussion above it is clear that to obtain the equivalent second-order formulation of NGT a Lagrange multiplier field $b_{\mu}$ must be introduced, and in fact the model equivalent to (2.6) is

$$
\mathcal{L}_{\mathrm{NGT}}^{(2)}(g, b)=\sqrt{-g} g^{\mu \nu} R_{\mu \nu}(\Gamma(g))-b_{\nu} \partial_{\mu}\left(\sqrt{-g} g^{[\mu \nu]}\right) .
$$

For, upon varying (2.19), we obtain

$$
R_{\mu \nu}+\partial_{[\mu} b_{\nu]}=0, \quad \partial_{\mu}\left(\sqrt{-g} g^{[\mu \nu]}\right)=0,
$$

from which we learn that

$$
\Gamma_{\mu \nu}^{\lambda}(g)=\widetilde{\Gamma}_{\mu \nu}^{\lambda}(g) .
$$

Thus the field equations are equivalent to those of the original first-order system, the Lagrange multiplier $b_{\mu}$ now taking the role formerly played by $\Gamma_{\mu}$ (more precisely, $b_{\mu}=\frac{-2}{D-1} \Gamma_{\mu}$ ). On the other hand, the simplest second-order theory, defined by Eq. (2.19) without the Lagrange multiplier [and introduced [12] under the name "algebraically extended Hilbert gravity" (AHG)], is

$$
\mathcal{L}_{\mathrm{AHG}}^{(2)}(g)=\sqrt{-g} g^{\mu \nu} R_{\mu \nu}(\Gamma(g)),
$$

with $\Gamma_{\mu \nu}^{\lambda}(g)$ determined by (2.18), and is very different from NGT. The consistency of both NGT and AHG will be critically examined below in Sec. IV.

By this point, the reader may have noted some pedantry in our introduction of the geometric theories and their equivalent formulations. Our motivation for this development was twofold. Firstly, to the extent that we will be obtaining no-go theorems, it is important to establish precisely what does not go. Secondly, the literature contains occasional confusing claims, for example, about inequivalence of first- and second-order formulations, so it also seems relevant to state exactly what we believe. At the risk of overkill, we will even give an explicit derivation of equivalence - for a model where it has been claimed otherwise, namely the linearizations of (2.6) and (2.19) -in Appendix B.

To conclude this "kinematical" section, we return to the fact that with a looser definition of "geometrical," one can write any generally covariant action involving the standard symmetric metric $G_{\mu \nu}$ and the matter field $B_{\mu \nu}$ in geometrical form: given any desired term, we may simply identify $G_{\mu \nu}$ and $B_{\mu \nu}$ as the symmetric and antisymmetric pieces of $g_{\mu \nu}$ respectively. Then one is left with the problem of representing the inverse $G^{\mu \nu}$ in terms of $g_{\mu \nu}$ and its inverse $g^{\mu \nu}$. Of course the penalty which one must pay is that an infinite number of terms will now be required in general to obtain this representation, so this constitutes far too loose a way to "unify" the fields. A somewhat more geometric rewriting of a generic term seems possible if one uses the fact that $g^{\mu \alpha} g_{\alpha \nu} \neq \delta_{\nu}^{\mu}$, which implies that a given string of terms built from the metric and its inverse-e.g., $g^{\mu \alpha} g_{\alpha \beta} g^{\beta \gamma} g_{\gamma \nu} \ldots$, with indices distributed as shown-will be nontrivial. [Indeed, a counting of parameters-at least to fourth order in the expansion-suggests that these strings may be used in combinations of curvature and torsion invariants to reproduce the desired term to any given order.]

\section{EXPANSION ABOUT A SYMMETRIC BACKGROUND}

In order to understand the consistency problems faced by generic geometric models, it is simplest to expand the field equations in powers of the $B_{\mu \nu}$ field about a general curved symmetric background $G_{\mu \nu}$. Because there has been some confusion about expandability [13], we emphasize that our analysis is based on the fact that the dynamics of these two separate components, $G_{\mu \nu}$ and $B_{\mu \nu}$, can be described by two independent coupling constants-namely the usual Einstein $\kappa^{2}$ and that associated with the antisymmetric field. We assume (as is standard in field theory) that expansion in these coupling constants is allowed, so that in particular the theory must reduce to Einstein gravity to zeroth order in the antisymmetric field, and require that it remain consistent ${ }^{2}$ order by order (without discontinuities in the degree-offreedom content). [For example, the generalization of the Schwarzschild solution in NGT is separately analytic in these two parameters and satisfies (4.5)-(4.7) below to 
the expected order in $B$.] Note that in general relativity there is a rigorous proof that the expansion in $\kappa^{2}$ is perfectly legal-even for studying the asymptotic behavior at infinity - in that it generates a series which is the Taylor expansion of some exact solution of the theory [15].

We will divide our analysis into two parts, according to whether the field equations are homogeneous of second-derivative order, or also include nonderivative"cosmological"-terms. Henceforth we work in secondorder formalism only, omitting the corresponding superscript 2 on Lagrangians.

\section{A. No "cosmological" terms}

The leading terms to consider in the action are those quadratic in $\partial B$. It is well known that (just as in electrodynamics) the only ghost-free action quadratic in a massless $B$ field is the square of its field strength (1.4), whatever the gravitational background:

$$
\mathcal{L}_{2}=-\frac{1}{12} \sqrt{-G} H_{\mu \nu \lambda} H^{\mu \nu \lambda},
$$

which is gauge invariant under (1.3). [We recall that all indices are moved by $G_{\mu \nu}$, our signature is mostly plus, and that the cyclic ordinary derivative ("curl") in the field strength (1.4) defines a tensor.] Hence any geometric action inequivalent (modulo field redefinitions) to (3.1) at order $(\partial B)^{2}$ already contains ghosts, and so cannot provide an acceptable effective action. [We shall see shortly that this conclusion holds even in the presence of apparent "gauge-fixing" terms, as in (2.19) for NGT.] The requirement (3.1), already necessary in flat space, is however far from sufficient when working about generic backgrounds. Consider, for example, a typical term in a geometric action (from Appendix A),

$$
\begin{aligned}
\sqrt{-g} g^{\mu \nu} R_{\mu \nu}(g)= & \sqrt{-G} \bar{R}(G)-\frac{1}{12} \sqrt{-G} H^{\mu \nu \lambda} H_{\mu \nu \lambda} \\
& +\frac{1}{2}\left[\frac{1}{2}-\alpha+(D-2) \beta\right] \sqrt{-G} \bar{R}(G) B^{2} \\
& -\alpha \sqrt{-G} \bar{R}_{\mu \nu}(G) B^{\mu \alpha} B_{\alpha}{ }^{\nu} \\
& -\sqrt{-G} \bar{R}_{\mu \nu \alpha \beta}(G) B^{\mu \alpha} B^{\nu \beta}+O\left(B^{4}\right)
\end{aligned}
$$

where bars denote covariantization with respect to the $G_{\mu \nu}$ background and we have dropped total derivative terms. In addition to the correct $(\partial B)^{2}$ terms (3.2) also contains (dimensionally equivalent) backgroundcurvature-coupled terms $\sim \bar{R} B B$. The latter fall into two classes: those involving only the Ricci tensor (or scalar) are removable by field redefinition, with the appropriate choice of $\alpha, \beta$ in (2.5). However, terms involving the full curvature cannot be so removed (for $D>3$ ). Their

\footnotetext{
${ }^{2}$ We underline that these generic nonlinearity expansions about a background are to be distinguished from the more specialized combined weak-field-short-wavelength (Isaacson) expansions [14]; contrary to the claims in [13] the latter have no particular relevance in the present context.
}

presence is fatal to the consistency of the theory because they destroy gauge invariance, which leads to unacceptable local constraints. This will be elaborated for the key cases in Sec. IV. It is clear from the formulas of Appendix $A$ that the consistency requirements at quadratic order in $B$ rule out almost all the second-order actions of the form (2.16). It is nevertheless possible to achieve a consistent action at quadratic order (pure $H^{2}$ without $\bar{R} B B$ terms) by combining several "geometric" terms. Using the results of Appendix A, an explicit example of a quadratically acceptable action is

$$
\begin{aligned}
\mathcal{L}=\sqrt{-g} g^{\mu \nu}[ & R_{\mu \nu}(g)-\Gamma_{\mu} \Gamma_{\nu}-T_{\mu \lambda}^{\alpha} T_{\alpha \nu}^{\lambda} \\
& \left.-\nabla_{\alpha} T_{\mu \nu}^{\alpha}-g_{\alpha \beta} g^{\rho \sigma} T_{\mu \rho}^{\alpha} T_{\nu \sigma}^{\beta}\right]
\end{aligned}
$$

after appropriate choice of field redefinition. Let us consider now the general class of quadratically acceptable actions,

$$
\mathcal{L}_{d}=\mathcal{L}_{2}+\sum_{n \geq 3} \mathcal{L}_{n} \equiv \mathcal{L}_{2}+\mathcal{L}_{>}
$$

Here $\mathcal{L}_{d}$ is a modification of the required gauge invariant $\mathcal{L}_{2}$ of (3.1) by higher-order $(n>2)$ terms in $B_{\mu \nu}$ (with two derivatives or a curvature term). Clearly, an expression of the form (3.4) is also not generally consistent since it involves terms of the form $\bar{R} B^{n}$ or $\partial B \partial B B^{n-2}$, so that it loses the initial invariance $\left(\delta_{0}\right)$ of $H_{\mu \nu \lambda}$. This loss of the local invariance entails unacceptable conservation constraints (notorious in higher spin gauge theories) on the nonlinear sources of the identically conserved linear term in the field equations. So, these models are only saved if the Abelian invariance itself can be deformed to

$$
\delta=\delta_{0}+\sum_{n \geq 1} \delta_{n} \equiv \delta_{0}+\delta_{>}
$$

such that the action $S_{d}$ is invariant under the deformed transformation. If such a deformation exists at all, it will also be present in particular when the background is flat, $G_{\mu \nu}=\eta_{\mu \nu}$. [The only exception would be a deformation of the full theory which reduced to the linear invariance at $G_{\mu \nu}=\eta_{\mu \nu}$. However, the deformation $\delta_{>}$must then be built with curvatures, and thus would contain too many derivatives to produce an invariance of a twoderivative order action.] We now show that there is no such deformation available even about a flat background (for an initial attempt at finding such a deformation, see the third reference cited in [12]).

We preface a demonstration of the nonexistence of deformations of (3.1) based on the technical approach of [16], by giving a transparent (though somewhat heuristic) argument for the case of Maxwell theory. In that case, one may ask for a consistent deformation of $\mathcal{L}^{M}(\partial A)=$ $-\frac{1}{4} F_{\mu \nu}^{2}$ by two-derivative terms $\mathcal{L}_{>}^{M}$ of higher order in $A_{\mu}$, invariant under the extension $\delta=\delta_{0}+\delta_{>}$with $\delta_{0} A_{\mu}=\partial_{\mu} \epsilon$. This was shown not to exist in [16]. Another way to see the problem is as follows. Let $\mathcal{L}_{n_{0}}, n_{0}>2$, be the first nonvanishing deformation; we then need $\delta_{n_{0}-2}$ with

$$
\delta_{0} S_{n_{0}}^{M}=-\delta_{n_{0}-2} S_{2}^{M} .
$$


But then

$$
\left.\partial_{\mu} \frac{\delta S_{n_{0}}^{M}}{\delta A_{\mu}}\right|_{0} \equiv \partial_{\mu} J^{\mu}=0
$$

where $\left.\right|_{0}$ means evaluated on free $\left(S_{2}^{M}\right)$ mass shell. Now if $J^{\mu}$ is a conserved current on linearized shell, there must (by Noether's theorem) be a corresponding continuous symmetry (with a scalar parameter $\tilde{\epsilon}$ say) of the Maxwell action, local of order $n_{0}-2$ in $A_{\mu}$ and of first-derivative order. (This ignores identically conserved "superpotential" currents $K^{\mu} \equiv \partial_{\nu} Z^{[\mu \nu]}$ which should be removed by field redefinitions.) Basically the statement is that there are none-and this may be verified by explicit enumeration (if nothing else) - except those equivalent to the original local gauge invariance,

$$
\widetilde{\delta} A_{\mu}=\widetilde{\epsilon} \partial_{\mu} f(A)
$$

whose Noether current is

$$
J^{\mu}=\left[\partial_{\nu} f(A)\right] F^{\mu \nu}(A)
$$

This is indeed a conserved current on linear shell, and in fact the field equation $\partial^{\nu} F_{\mu \nu}=\left[\partial^{\nu} f(A)\right] F_{\mu \nu}$ is perfectly consistent, though not gauge invariant. However, it cannot be derived from an action; thus one cannot find any consistent deformations of the Maxwell action and its gauge invariance. This argument may probably be extended to the $B$ field, but for technical ease we will follow [16]. Going back to our $B_{\mu \nu}$ problem, (3.4), let us begin with a quick review of the main points in the approach of [16]. (Note that we do not derive here the most general result possible, but simply demonstrate it in the context of actions homogeneous in two derivatives as is relevant to our discussion.) Denote the equation of motion by $\mathcal{F}^{\mu \nu}$,

$$
\mathcal{F}^{\mu \nu}=\frac{\delta S_{d}}{\delta B_{\mu \nu}}=\mathcal{F}_{1}^{\mu \nu}+\mathcal{F}_{2}^{\mu \nu}+\cdots,
$$

where $\mathcal{F}_{1}^{\mu \nu}=\frac{1}{2} \partial_{\lambda} H^{\lambda \mu \nu}$, and the "linearized Bianchi identity" is

$$
\partial_{\mu} \mathcal{F}_{1}^{\mu \nu} \equiv 0
$$

For the deformed theory to preserve its degree-of-freedom content, the field equations (3.10) must admit a corresponding (nonlinear) Bianchi identity, which can be written as

$$
\begin{aligned}
\partial_{\mu}\left(U_{\alpha \beta}^{\mu \nu} \mathcal{F}^{\alpha \beta}\right) & =U_{\alpha \beta}^{\mu \lambda} V_{\mu \lambda}^{\nu} \mathcal{F}^{\alpha \beta}, \\
U_{(0) \alpha \beta}^{\mu \nu} & =\delta_{[\alpha}^{\mu} \delta_{\beta]}^{\nu}, V_{(0) \alpha \beta}^{\lambda}=0 .
\end{aligned}
$$

In turn, the identity (3.12) is equivalent to requiring invariance of the action $S_{d}$ under the deformed gauge transformation

$$
\delta B_{\mu \nu}=U_{\mu \nu}^{\alpha \beta}\left(\partial_{\alpha} \epsilon_{\beta}+V_{\alpha \beta}^{\lambda} \epsilon_{\lambda}\right)
$$

Here, consistent with the derivative constraints, $U_{\alpha \beta}^{\mu \nu}=$ $-U_{\beta \alpha}^{\mu \nu}$ is algebraic in $B_{\mu \nu}$ and $V_{\alpha \beta}^{\lambda}$ contains at most one derivative of $B_{\mu \nu}$. Clearly this is a redundant parametrization, in that $(U, V)$ is equivalent to the set
$\left(U^{\prime}, V^{\prime}\right)$ for which there is a matrix $f$ algebraic in $B_{\mu \nu}$, $f_{(0) \mu}^{\nu}=\delta_{\mu}^{\nu}$, such that

$$
\begin{aligned}
& \left(U^{\prime}\right)_{\alpha \beta}^{\mu \nu}=f_{\gamma}^{\nu} U_{\alpha \beta}^{\mu \gamma} \\
& \left(V^{\prime}\right)_{\alpha \beta}^{\lambda}=f_{\delta}^{\lambda} V_{\alpha \gamma}^{\delta}\left(f^{-1}\right)_{\beta}^{\gamma}+\left(f^{-1}\right)_{\beta}^{\gamma} \partial_{\alpha} f_{\gamma}^{\lambda} .
\end{aligned}
$$

Further, one easily sees that a field redefinition only affects $U$, and that there exists (locally) a field redefinition which sets $U_{\alpha \beta}^{\mu \nu}=\delta_{[\alpha}^{\mu} \delta_{\beta]}^{\nu}$ if and only if

$$
\frac{\partial U_{\alpha \beta}^{\mu \nu}}{\partial B_{\gamma \delta}} U_{\gamma \delta}^{\rho \sigma}-\frac{\partial U_{\alpha \beta}^{\rho \sigma}}{\partial B_{\gamma \delta}} U_{\gamma \delta}^{\mu \nu}=0 .
$$

One obtains constraints on the existence of a deformed gauge invariance of the form (3.13) by demanding the closure of the gauge algebra, namely that it be possible to find a transformation parameter $\epsilon^{\prime \prime}$ (depending on $\left.\epsilon, \epsilon^{\prime}, B\right)$ such that

$$
\left[\delta, \delta^{\prime}\right]=\delta^{\prime \prime} \text {. }
$$

The strategy is to try to determine $U$ and $V$ order by order in $B_{\mu \nu}$, by imposing (3.16). At the first order we have candidates (the symmetric part of $V$ at this order will be seen to be irrelevant)

$$
\begin{gathered}
U_{(1) \alpha \beta}^{\mu \nu}=b_{1} B_{[\alpha}^{\mu} \delta_{\beta]}^{\nu}+b_{2} B_{[\alpha}^{\nu} \delta_{\beta]}^{\mu}, \\
V_{(1)[\alpha \beta]}^{\lambda}=a_{1} \partial^{\lambda} B_{\alpha \beta}+a_{2} \partial_{[\alpha} B_{\beta]}^{\lambda}+a_{3} \partial^{\gamma} B_{\gamma[\alpha} \delta_{\beta]}^{\lambda} .
\end{gathered}
$$

By using the freedom (3.14), with $f_{(1) \mu}{ }^{\nu} \sim B_{\mu}{ }^{\nu}$, we may set $b_{1}=-b_{2}$. To zeroth order these are all that are required in (3.16), for which at this order the right-hand side is just $\partial_{[\mu} \epsilon_{(0) \nu]}^{\prime \prime}$. Thus we may simplify by taking $\partial_{\lambda}$ and totally antisymmetrizing over $[\lambda \mu \nu]$, to obtain

$$
\begin{gathered}
-2 b_{2}\left[\partial_{[\mu} \epsilon^{\alpha} \partial_{\lambda} \partial_{\alpha} \epsilon_{\nu]}^{\prime}-\partial_{[\lambda} \partial^{\alpha} \epsilon_{\mu} \partial_{\alpha} \epsilon_{\nu]}^{\prime}-\left(\epsilon \leftarrow \rightarrow \epsilon^{\prime}\right)\right] \\
+\left(a_{1}-a_{2}\right)\left[\partial^{\alpha} \partial_{[\mu} \epsilon_{\nu} \partial_{\lambda]} \epsilon_{\alpha}^{\prime}-\left(\epsilon \leftarrow \rightarrow \epsilon^{\prime}\right)\right] \\
+a_{3}\left[\square \partial_{[\lambda} \epsilon_{\mu} \epsilon_{\nu]}^{\prime}+\square \epsilon_{[\mu} \partial_{\lambda} \epsilon_{\nu]}^{\prime}\right. \\
\left.-\partial_{[\mu} \partial^{\alpha} \epsilon_{\alpha} \partial_{\lambda} \epsilon_{\nu]}^{\prime}-\left(\epsilon \leftarrow \rightarrow \epsilon^{\prime}\right)\right]=0 .
\end{gathered}
$$

Setting $\epsilon_{\mu}$ to be a constant vector we learn that $a_{3} \square \partial_{[\lambda} \epsilon_{\mu}^{\prime} \epsilon_{\nu]}=0$ for all $\epsilon^{\prime}$, and thus $a_{3}=0$. Similarly, taking $\partial_{\mu} \epsilon_{\nu}=S_{\mu \nu}=S_{\nu \mu}$ constant, and $\partial_{\mu} \epsilon_{\nu}=A_{\mu \nu}=-A_{\nu \mu}$ constant, we find $b_{2}=0$ and $a_{1}=a_{2}$. Thus imposing the closure constraint (3.16) to zeroth order in $B$ has reduced the first-order deformation to

$$
\begin{gathered}
U_{(1) \alpha \beta}^{\mu \nu}=0, \\
V_{(1)[\alpha \beta]}^{\lambda}=a_{1} H_{\alpha \beta}^{\lambda} .
\end{gathered}
$$

Moreover, inserting (3.19) into (3.16) yields $\partial_{[\mu} \epsilon_{(0) \nu]}^{\prime \prime}=$ 0 , from which we deduce that $\epsilon_{(0) \mu}^{\prime \prime}=0$ because it is impossible to construct a pure gradient which is bilinear in $\epsilon_{\mu}$ and $\epsilon_{\mu}^{\prime}$ and antisymmetric under their exchange. If we now consider the special case for which $\epsilon_{\mu}=\partial_{\mu} \epsilon$ 
and $\epsilon_{\mu}^{\prime}=\partial_{\mu} \epsilon^{\prime}$, then (3.19) is all we require to impose (3.16) to first order since the zeroth-order variation of $B$ vanishes. The right-hand side of (3.16) then reduces at lowest order to $\partial_{[\mu} \epsilon_{(1) \nu]}^{\prime \prime}$, where $\epsilon_{(1) \nu}^{\prime \prime}$ is linear in $B$. As above, on taking the curl of the resulting equation we obtain a simple constraint which can generically only be satisfied if $a_{1}$ vanishes. Another way to prove that necessarily $a_{1}=0$ is to check that there is no combination of the two possible nontrivial terms in a cubic action,

$$
\mathcal{L}_{3}=B_{\mu \nu}\left(A_{1} \partial^{\mu} B^{\nu \beta} \partial^{\alpha} B_{\alpha \beta}+A_{2} \partial^{\mu} B_{\alpha \beta} \partial^{\alpha} B^{\nu \beta}\right),
$$

which can compensate the change of (3.1) under $\delta_{1} B_{\mu \nu}=$ $H^{\lambda}{ }_{\mu \nu} \epsilon_{\lambda}$, i.e., which solves $\delta_{1} \mathcal{L}_{2}=-\delta_{0} \mathcal{L}_{3}$. Having proven the triviality of all possible first-order deformations, an inductive argument using (3.16) exactly as in [16] shows that

$$
\begin{gathered}
U_{(n) \alpha \beta}^{\mu \nu}=0, n \geq 1, \\
V_{(n)[\alpha \beta]}^{\lambda}=0, n \geq 0 .
\end{gathered}
$$

Hence we have shown that it is not possible to produce a deformation which will give a consistent generalization of the gauge invariance in this case. As the only gauge invariant action ${ }^{3}$ that can be written down in $D=4$ is $\int d^{4} x \sqrt{-G} H^{2}$, we conclude that (3.1) defines the only consistent theory in the absence of cosmological terms.

We now briefly return to close the remaining loophole, that perhaps some terms beyond (3.1) appearing at quadratic order could be interpreted as "gauge fixing." The main point here is that the very notion of gauge fixing does not make sense unless there is an underlying gauge-invariant theory,-and, as we have just shown, there is no natural candidate in this geometric context. Therefore all the Lagrangians (2.16) containing $\Gamma_{\mu}$ multipliers will necessarily exhibit propagating ghosts, without the possibility of projecting onto an invariant ghost-free subspace. It is here that the lack of underlying gauge invariance is lethal-this moral is well known, and we content ourselves with illustrating it in an example in Sec. IV.

\section{B. With "Cosmological" terms}

Suppose we now also permit "cosmological terms" (i.e., terms without derivatives) in the Lagrangian. The archetypal example is $\sqrt{-g}=\sqrt{-G}\left(1+\frac{1}{4} B^{2}+\cdots\right)$, but we could also arrange to cancel $\sqrt{-G}$ or in general give different coefficients to $\sqrt{-G}$ and the $B^{2}$ term (see Appendix A). Perhaps surprisingly, this alters the no-go conclusions of the preceding subsection despite the fact that $\sqrt{-g}$ is certainly geometric in the narrowest sense. [As emphasized by Schrödinger, who favored them [3], such models can even be obtained from the geometrically

\footnotetext{
${ }^{3}$ Note however the possibility, when $D=5$, of ChernSimons-type kinetic terms: $\int d^{5} x \epsilon^{\mu \nu \lambda \alpha \beta} H_{\mu \nu \lambda} B_{\alpha \beta}$.
}

elegant-purely affine-Lagrangian $\left.\sqrt{-\operatorname{det} \mathrm{R}_{\mu \nu}(\Gamma)}.\right]$ The point is that once a mass term $\left(\sim-\frac{1}{4} m^{2} B^{2}\right)$ is included, then the obvious constraints associated with gauge invariance disappear; for example, we do not need to attempt the (fruitless) deformation route to avoid conservation inconsistencies. [Of course, the second-derivative quadratic term must still be $H^{2}$ for ghost-freedom, just as it is only $-\frac{1}{4} F^{2}-\frac{1}{2} m^{2} A^{2}$, but not $\frac{1}{2} A^{\mu}\left(\square-m^{2}\right) A_{\mu}$ which is an acceptable finite-range vector Lagrangian.] As an illustrative example, consider the Lagrangian

$\mathcal{L}=-\frac{1}{12}\left(1+f_{1} B^{2}\right) H_{\lambda \mu \nu}^{2}-\frac{1}{4} m^{2} B^{2}-f_{2} B^{2} B^{2}$,

where $f_{i}$ are some coupling constants. The equations of motion are

$$
\begin{array}{r}
\frac{1}{2} \partial_{\lambda}\left[\left(1+f_{1} B^{2}\right) H^{\lambda \mu \nu}\right]-\frac{1}{6} f_{1} H^{2} B^{\mu \nu}-\frac{1}{2} m^{2} B^{\mu \nu} \\
-4 f_{2} B^{2} B^{\mu \nu}=0 .
\end{array}
$$

We may look for a solution expanded in "powers of nonlinearity," $B_{\mu \nu}=\sum_{n \geq 0} B_{\mu \nu}^{(n)}$ around $B^{(0)}=0$. Up to third order, the equations are

$$
\begin{aligned}
& \frac{1}{2} \partial_{\lambda} H^{\lambda \mu \nu}\left(B^{(1)}\right)-\frac{1}{2} m^{2}\left(B^{(1)}\right)^{\mu \nu}=0 \\
& \frac{1}{2} \partial_{\lambda} H^{\lambda \mu \nu}\left(B^{(2)}\right)-\frac{1}{2} m^{2}\left(B^{(2)}\right)^{\mu \nu}=0, \\
& \frac{1}{2} \partial_{\lambda} H^{\lambda \mu \nu}\left(B^{(3)}\right)-\frac{1}{2} m^{2}\left(B^{(3)}\right)^{\mu \nu} \\
& \quad+\frac{1}{2} \partial_{\lambda}\left[f_{1}\left(B^{(1)}\right)^{2} H^{\lambda \mu \nu}\left(B^{(1)}\right)\right]-4 f_{2}\left(B^{(1)}\right)^{2}\left(B^{(1)}\right)^{\mu \nu} \\
& \quad-\frac{1}{6} f_{1}\left[H\left(B^{(1)}\right)\right]^{2} B^{(1) \mu \nu}=0 .
\end{aligned}
$$

As long as $m^{2} \neq 0$ there is no problem with consistency constraints from lower-order equations on the higherorder ones. For example, now using the identity (3.11) simply determines that $\partial^{\mu} B_{\mu \nu}^{(1)}=0=\partial^{\mu} B_{\mu \nu}^{(2)}$ and gives a relation for $\partial^{\mu} B_{\mu \nu}^{(3)}$ in terms of the lower-order terms in the solution.

Although $m^{2} \neq 0$ avoids the local consistency constraints in a perturbative expansion in $B_{\mu \nu}$, more work would be needed to check whether, from a mathematical standpoint, the field equations associated with say (3.22) form a well-posed problem, with acceptable causality properties.

\section{PROBLEMS WITH THE BASIC MODELS}

In light of our discussion in Sec. III A, it should be clear that a generic (massless) geometric model will violate either ghost-freedom or local consistency requirements. In this section, we will illustrate these failings by studying in some detail the two main examples of geometric models which have been proposed in the literature. [12],

Consider first the simplest second-order theory (AHG)

$$
\mathcal{L}_{\mathrm{AHG}}^{(2)}(g)=\sqrt{-g} g^{\mu \nu} R_{\mu \nu}(g) .
$$

As we saw from (3.2), this model's expansion already has a term quadratic in $B_{\mu \nu}$, proportional to the full Riemann tensor. It can neither be field redefined away, nor is it $\delta_{0}$-gauge invariant. Thus the model is sick already at quadratic order, having "ghost" kinematics, 
even though its flat space limit is the required $H^{2}$ theory. This much was noticed in [17]; earlier it had separately been noted that these theories had no asymptotically flat static spherically symmetric solution [18]. To elucidate further the sickness of the model when expanded on a curved background, let us consider the field equations, which decompose into symmetric and antisymmetric pieces as follows:

$$
\begin{aligned}
& \bar{R}_{\mu \nu}+O\left(B^{2}\right)=0, \\
& \bar{\nabla}^{\alpha} H_{\alpha \mu \nu}-4 \bar{R}_{\mu \alpha \nu \beta} B^{\alpha \beta}+O\left(B^{3}\right)=0 .
\end{aligned}
$$

As we are only looking for a family of solutions continuously connected to those of general relativity, we can ignore the terms of higher order in $B$ in these equations. By the identical conservation of $\bar{\nabla} H$, we then have the consistency requirement

$$
\bar{\nabla}^{\mu} \bar{R}_{\mu \alpha \nu \beta} B^{\alpha \beta}+\bar{R}_{\mu \alpha \nu \beta} \bar{\nabla}^{\mu} B^{\alpha \beta}=0,
$$

which by the Bianchi identities (using $\bar{R}_{\mu \nu}=0$ ) can be simplified to

$$
\bar{R}_{\mu \alpha \nu \beta} \bar{\nabla}^{\mu} B^{\alpha \beta}=0 .
$$

This is an unacceptable local constraint on the "independent" degrees of freedom.

Our second example is NGT, which has been regarded as the remaining geometric model apparently not disqualified by previous work. Its linearization about flat space, considered in Appendix B, consists of linearized Einstein theory plus a gauge fixed version of the $H^{2}$ action [7]. One might therefore have hoped that its undesirable excitations could always be projected away in a consistent fashion, and thus it would exemplify "geometric theories with built-in gauge fixing," as discussed at the end of Sec. III A. We now show, however, that NGT is inconsistent at the next level where the $G$ background is no longer flat. ${ }^{4}$ Thus the analogy to gauge fixing will be seen to be invalid, consistent with our previous general conclusions. (The following discussion amplifies on the result briefly announced in [11], and is included for completeness.)

Using (A8), the field equations, to zeroth and linear order in $B_{\mu \nu}$, become

$$
\begin{aligned}
\bar{R}_{\mu \nu} & =0 \\
\bar{\nabla}^{\lambda} H_{\lambda \mu \nu}-4 \bar{R}_{\mu \nu}^{\alpha{ }^{\beta} B_{\alpha \beta}} & =\frac{4}{D-1} \partial_{[\mu} \Gamma_{\nu]}, \\
\bar{\nabla}^{\mu} B_{\mu \nu} & =0
\end{aligned}
$$

upon inserting the solution for $\widetilde{\Gamma}$ to this order into the $g_{\mu \nu}$ field equations. In a flat background, this system of course reduces to the set (B5), (B6), which is shown there to be precisely a gauge fixed system. The gauge condition (B6) still admits a residual gauge invariance,

\footnotetext{
${ }^{4}$ The inconsistency to be exhibited is quite different from that exemplified by AHG. There are no analogues in NGT of the local algebraic inconsistencies of the type (4.4), as is implicit in the mathematical study [4] of the local analytic Cauchy problem for NGT; its problems are of a physical and global nature.
}

namely $\delta B_{\mu \nu}=\partial_{[\mu} \epsilon_{\nu]}$ with $\partial^{\mu} \partial_{[\mu} \epsilon_{\nu]}=0$. If we consider instead a generic Ricci-flat background (4.5), then the putative residual gauge invariance

$$
\delta B_{\mu \nu}=\partial_{[\mu} \epsilon_{\nu]}, \quad \bar{\nabla}^{\mu} \bar{\nabla}_{[\mu} \epsilon_{\nu]}=0
$$

is clearly lost because of the $\bar{R} B$ term in Eq. (4.6). This is an immediate indication that the system in a generic background is no longer the gauge-fixed version of a ghost-free theory. (A contrary statement in [17] has since been corrected in [19].)

A more direct way to exhibit the problems of the system (4.5)-(4.7) consists of taking the divergence of (4.6). This yields

$$
\bar{\nabla}^{\mu} \bar{\nabla}_{[\mu} \Gamma_{\nu]}=-(D-1) \bar{R}_{\mu}^{\alpha{ }_{\nu}{ }^{\beta}} \bar{\nabla}^{\mu} B_{\alpha \beta},
$$

which is an inhomogeneous Maxwell equation for $\Gamma_{\mu}$. Consequently, one can no longer fix initial conditions to eliminate $\Gamma_{\mu}$ invariantly, and these dangerous extra modes do not decouple, even in the vacuum theory we are considering here. The situation would be worse in any matter-coupled version of the theory as the righthand side of (4.9) would contain additional terms acting as localized sources of retarded $\Gamma_{\mu}$ waves.

We now show how the existence of a propagating $\Gamma_{\mu}$ implies the presence of ghost (negative energy) modes. To be explicit, let us first summarize how energy is to be calculated in the theory, or more relevantly, in our expansion scheme. For simplicity, we consider the problem in $D=4$ spacetime and we treat the case in which there is no normal cosmological term $\sqrt{-G}$; its presence would not alter our conclusions. Then, we require asymptotic flatness, and hence sufficiently rapid decay of the $B$ field at infinity. This permits us to split the action through order $B^{2}$ into "Einstein plus matter" form:

$$
\begin{aligned}
I=\int d^{4} x \sqrt{-G}\left[\bar{R}-\frac{1}{12} H^{2}-\frac{2}{3}\left(\partial_{\mu} \Gamma_{\nu}-\partial_{\nu} \Gamma_{\mu}\right) B^{\mu \nu}\right. \\
\left.\quad-\bar{R}_{\mu \alpha \nu \beta} B^{\mu \nu} B^{\alpha \beta}+O\left(B^{3}\right)\right] \\
\equiv \int d^{4} x \sqrt{-G} \bar{R}+I_{M} .
\end{aligned}
$$

In this formulation the matter stress tensor reads

$$
\begin{aligned}
T_{M}^{\mu \nu}= & 2(-G)^{-1 / 2} \frac{\delta}{\delta G_{\mu \nu}} I_{M} \\
= & \left(\frac{1}{2} H^{\mu \alpha \beta} H_{\alpha \beta}^{\nu}-\frac{1}{12} G^{\mu \nu} H^{2}\right) \\
& +\left(\frac{4}{3} B^{\mu \alpha} f_{\alpha}^{\nu}+\frac{4}{3} B^{\nu \alpha} f_{\alpha}^{\mu}-\frac{2}{3} G^{\mu \nu} f^{\alpha \beta} B_{\alpha \beta}\right) \\
& +\left(3 \bar{R}_{\gamma \alpha \beta}^{(\mu} B^{\nu) \alpha} B^{\gamma \beta}-G^{\mu \nu} \bar{R}_{\gamma \alpha \delta \beta} B^{\gamma \delta} B^{\alpha \beta}\right) \\
& -\bar{\nabla}_{\beta} \bar{\nabla}_{\alpha}\left(B^{\alpha(\mu} B^{\nu) \beta}\right),
\end{aligned}
$$$$
f_{\mu \nu} \equiv \partial_{\mu} \Gamma_{\nu}-\partial_{\nu} \Gamma_{\mu}
$$

Only the first term on the right-hand side leads to positive energy; its 00 component is (omitting metric factors) given by $\sim 3 H_{0 i j}^{2}+H_{i j k}^{2}$. The remaining ones fail to do so, even after using the $B$ equations of motion (4.6) to eliminate $f_{\mu \nu}=\frac{3}{4}\left(\bar{\nabla}^{\lambda} H_{\lambda \mu \nu}-4 \bar{R}_{\mu \alpha \nu \beta} B^{\alpha \beta}\right)$. Thus the proof of positivity of energy for normal fields fails here. Still assuming that all fields fall off sufficiently fast at 
infinity, let us consider the asymptotic energy flux associated with (4.11). At infinity, the third term in (4.11) vanishes, while the fourth one is easily seen to be a simple superpotential (i.e., of the form $\partial_{\alpha} \partial_{\beta} K^{[\mu \alpha][\nu \beta]}$ ) making no net contribution to the energy-momentum flux. On the other hand, the first term in (4.11) gives a positive contribution corresponding to the radiation of the one scalar mode contained in the transverse projection of $B_{i j}$, while the second term gives a nonpositive contribution associated with the interplay between the radiation of $B_{0 i}$ and that of $\Gamma_{i}$. When comparing our results with those of the related analysis in [20], we found sign mistakes between (2.21c) of [20] and the "NGT luminosity formula" (3.16) of [20]. In fact, the signs of the NGT terms in (3.16) of [20] should be reversed to be made to agree with our (4.11). With corrected signs the results (4.39) for the "radiation fields to lowest post-Newtonian order" in [20] imply that NGT waves radiate a negative flux of dipole radiation. However it must be pointed out that such a calculation is even in itself undermined by another dire physical consequence of NGT, namely the loss of any acceptable falloff behavior of $B_{\mu \nu}$ in the wave zone.

To investigate the fall-off behavior, let us assume that the curvature decays fast enough at infinity. ${ }^{5}$ Then, (4.9) yields (in the Lorentz gauge $\bar{\nabla}^{\mu} \Gamma_{\mu}=0$ ) an inhomogeneous wave equation for $\Gamma_{\mu}$ which implies that it has the usual $1 / r$ falloff at future null infinity. However, inserting this information in the right-hand side of (4.6), one finds that $B_{\mu \nu}$ (and in particular its longitudinal part) fails to vanish at future null infinity. For example, we have checked explicitly that in the usual matter-coupled version of NGT [7], considered in the post-Newtonian approximation [20], $\Gamma_{\mu}$ is predominantly radiated as a dipole, i.e.,

$$
\begin{aligned}
& \Gamma^{0} \sim-\partial_{i}\left[\partial_{t}^{2} D^{i}(t-r) / r\right] \\
& \Gamma^{i} \sim \partial_{t}^{3} D^{i}(t-r) / r
\end{aligned}
$$

where $D^{i}$ is the dipole moment of the conserved particle number density $S^{0}$ used as a macroscopic source in NGT. This in turn generates a nondecaying behavior for $B_{\mu \nu}$, e.g., for $r \rightarrow \infty$ at constant $t-r$,

$$
\begin{aligned}
& B_{0 i}(\mathbf{r}, t)=A\left(n_{i} n_{j}-\delta_{i j}\right) \partial_{t}^{3} D^{j}(t-r)+O(1 / r) \\
& B_{i j}(\mathbf{r}, t)=A n_{[i} \partial_{t}^{3} D_{j]}+O(1 / r)
\end{aligned}
$$

where $n^{i} \equiv \frac{r^{i}}{r}$, and $A$ is a constant. The problem can only get worse at higher orders in $B_{\mu \nu}$, suggesting that the fully nonlinear theory in $B$ does not make sense globally either.

\footnotetext{
${ }^{5}$ This assumption permits us to treat the source terms in (4.9) as quasilocalized, and hence is clearly the most favorable choice. Any weakening of this assumption would generically lead to worse fall-off behavior and could only strengthen our negative conclusions below.
}

There have been recent preprints $[13,21]$ which disagree with our conclusions. The claims in these preprints, beyond those we have explicitly dealt with above, seem to be of two types: that full nonperturbative solutions of NGT would not exhibit the pathologies we have found in our expansion; and that the bad asymptotic behavior of $B_{\mu \nu}$ is a consequence of the use of the Lagrange multipliers $\Gamma_{\mu}$. The latter is easily dealt with by eliminating the Lagrange multipliers and showing that the resulting equations reproduce the bad asymptotic behavior. ${ }^{6}$ The former is in general outside the scope of this paper. It would be extremely interesting to find such solutions. In [21] an axisymmetric solution was proposed as a modification of the Bondi soultion in general relativity. Unfortunately, one can quite straightforwardly see that this proposal does not satisfy all the field equations of NGT. In the first version of [21] their antisymmetric field equation (4.4f) is an ordinary differential equation for the function of one variable $\alpha(u)$ with coefficients also depending on the other variables $r$ and $\theta$. Therefore, in the general case of an arbitrary general relativistic "news function" $c(u, \theta)$, it admits only the trivial solution $\alpha(u)=0$. The June 1992 revision of [21] contains essentially the same error as the original version: their new ansatz again fails to satisfy all the required field equations. Specifically, the antisymmetric Ricci components of their (4.11) do not obey their antisymmetric field equation (4.1). These conclusions also follow from considering the leading terms in the Bondi expansion of our field equation (4.6) above, as we have explicitly checked. Beyond these specific problems, one would surely also expect-even on phenomenological groundsthat the most important sector in the solution space is precisely that smoothly connected to the solutions of general relativity. In any case, we feel that to find a pathology in this sector is to find a serious defect in the theory.

Let us note that the above inconsistencies are reminiscent of the well-known problems with higher spin mattergravity couplings (see, e.g., [22]). Like the latter they occur at the first nontrivial level beyond flat space, and give rise to complicated causality structure. Indeed, it is clear from the presence of the $\bar{R}_{\mu \nu \alpha \beta} B^{\mu \alpha} B^{\nu \beta}$ term in the action, which couples second derivatives of $G_{\mu \nu}$ with $B^{2}$ terms, that the characteristics of the theory will differ from the usual (multiple) $G_{\mu \nu}$-light cone by $B^{2}$ terms. In fact, mathematical study of the exact characteristics of vacuum NGT $[4,6]$, showed the existence of three dif-

\footnotetext{
${ }^{6}$ First, taking the curl of (4.6) eliminates $\Gamma_{\mu}$, leaving an inhomogeneous wave equation for $H_{\lambda \mu \nu}$. At best, then, $H$ decays as $1 / r$ at null infinity. Next, we determine $B_{\mu \nu}$ from (4.7) and the definition (1.4) of $H$ : Rewriting the latter two equations in terms of the duals $\left(F^{\mu \nu}, J^{\lambda}\right)$ of $\left(B_{\alpha \beta}, H_{\alpha \beta \gamma}\right)$ respectively, leads to ordinary Maxwell equations for the field $F^{\mu \nu}$ with source $J^{\lambda}$. Since the "current" $J$, i.e., $H$, falls off as $1 / r$, we have thereby confirmed-without having had recourse to $\Gamma_{\mu}$-the conclusion that $F$, i.e., $B$, generically fails to decay at the null infinity.
} 
ferent characteristic cones along which coupled $G-B$ modes propagate.

In summary, we conclude that since only $\bar{R}(G)+H^{2}$ is consistent, and all natural two-derivative "geometric" formulations typically have both unremovable naked $B$ dependence and/or the wrong $(\bar{\nabla} B)^{2}$ structure, Pauli's words about NGT, "what God has put asunder, let no man set together" apply quite aptly to all nonsymmetric gravity models without cosmological terms.

\section{FINITE-RANGE MODELS}

We saw in the previous sections that "geometric" nonsymmetric theories generically failed to obey consistency requirements unless the antisymmetric field is given a finite range. ${ }^{7}$ In this section we consider these massive models with emphasis on their possible observational consequences when coupled with matter, exploring ways in which the antisymmetric tensor contributes to the effective gravitational interactions of matter at laboratory or celestial scales. Needless to say, we will keep to ghost-free models whose kinematic terms are the gauge invariant $-\frac{1}{12} H_{\lambda \mu \nu}^{2}$. To set some bounds on the range $\mu^{-1}$ (or mass $\mu$ ), which is a free parameter, we recall that its formal introduction was through a cosmological-type term which suggests an upper bound $\mu^{-1} \sim \Lambda^{-1 / 2} \sim H_{0} \sim 10^{10}$ light years. A lower bound will be dictated by the matter couplings chosen and the limitations due to technical possibilities.

The basic question in studying alternative gravity models is to identify plausible macroscopic sources of $B_{\mu \nu}$. The first possibility that suggests itself is a direct coupling of $B_{\mu \nu}$ to some kind of microscopic polarization tensor, say $J^{\mu \nu}=\bar{\psi} \sigma^{\mu \nu} \psi$. However, such a coupling yields negligible macroscopic forces between ordinary (unpolarized) laboratory or celestial bodies, and therefore does not constitute an interesting starting point for our purposes. A different possibility is suggested by the link with the geometrical $g_{\mu \nu}-\Gamma_{\mu \nu}^{\lambda}$ models explored above. Indeed, Eq. (A5) for instance shows that the models we want to explore contain a propagating torsion

$$
T_{\mu \nu}^{\lambda}=\bar{\nabla}^{\lambda} B_{\mu \nu}-\frac{1}{2} H_{\mu \nu}^{\lambda}+O\left(B^{3}\right) .
$$

By analogy with the coupling of $G_{\mu \nu}$ to the stress-energy tensor $T^{\mu \nu}$, it is natural to look for a 3-index macroscopic source $S_{\lambda}^{\mu \nu}$ coupled to the torsion (5.1) —or, by duality, a vector current coupled to $\epsilon_{\lambda \alpha}^{\mu \nu} T_{\mu \nu}^{\lambda}$. Natural candidates are Dirac currents, $\bar{\psi} \gamma_{\alpha} \psi$ (the resulting coupling constant is dimensionless if $B_{\mu \nu}$, like $G_{\mu \nu}$, is geometrically normalized). Let us denote a general combination of such fermion currents by $J^{\mu}$. The total antisymmetry of the tensor density $J^{* \lambda \mu \nu} \equiv \epsilon^{\lambda \mu \nu \alpha} J_{\alpha}$ implies that its coupling

\footnotetext{
${ }^{7}$ We noted that the symmetric part of the field need not itself acquire a cosmological constant; in any case the latter is not relevant in the search for distinctly non-Einsteinian effects.
}

to the torsion (5.1) involves only the field strength $H_{\lambda \mu \nu}$. (Note that throughout the remainder of this section the $\epsilon$ symbols will be simply a totally antisymmetric collection of numerical constants, completely metric independent. Thus $\epsilon^{\lambda \mu \nu \alpha}$ transforms as a contravariant tensor density, while $\epsilon_{\lambda \mu \nu \alpha} \equiv \epsilon^{\lambda \mu \nu \alpha}= \pm 1,0$ is not related by the metric. When needed, appropriate factors of $\sqrt{-G}$ will be inserted explicitly.)

The basic macroscopic coupling of the $B$ field that we shall consider is then

$$
\begin{aligned}
S_{J}=\int d^{4} x \mathcal{L}_{J} & =\frac{-1}{6} f \int d^{4} x H_{\lambda \mu \nu} J^{* \lambda \mu \nu} \\
& =\frac{1}{2} f \int d^{4} x \epsilon^{\mu \nu \alpha \beta} B_{\alpha \beta} \partial_{\mu} J_{\nu},
\end{aligned}
$$

where $f$ is dimensionless if $B_{\mu \nu}$ is. The Pauli coupling (5.2), being actually gauge invariant for any $J_{\nu}$ (conserved or not), could also have been used to couple a massless (gauge) $B$ field to matter. However, it is easily seen that in that context $B_{\mu \nu}$ is really a massless scalar, $\phi$, coupled to $\partial_{\mu} J^{\mu}$, which is merely another way of introducing an extra scalar interaction.

Having selected a basic macroscopic source for the $B$ field, we can use the geometric motivation to add further phenomenological couplings between matter and the $B$ field. In particular, using the general field redefinition (2.5), we can define a class of metric theories of gravity where matter also feels the influence of a two-parameter "physical" metric

$$
\tilde{g}_{\mu \nu}=G_{\mu \nu}+a B_{\mu \alpha} B_{\nu}^{\alpha}+b B_{\alpha \beta} B^{\alpha \beta} G_{\mu \nu}+O\left(B^{4}\right) .
$$

Drawing on the example of the Einstein-Cartan-type theories, with independent torsion, propagating or not (see, e.g., [23]), we can also consider more general theories where matter is coupled to the above $\tilde{g}_{\mu \nu}$, as well as to some independent connection with torsion, say

$$
\hat{\Gamma}_{\mu \nu}^{\lambda}=\left\{\begin{array}{l}
\lambda \\
\mu \nu
\end{array}\right\}_{\tilde{g}}+c \widetilde{\nabla}^{\lambda} B_{\mu \nu}+d \tilde{g}^{\lambda \alpha} H_{\alpha \mu \nu}+\cdots,
$$

where the first term on the right-hand side denotes the Christoffel connection of $\tilde{g}_{\mu \nu}$ and where $c$ and $d$ are two additional parameters. Further terms could represent similar torsion terms built with the dual of $B_{\mu \nu}$, and/or higher-order terms.

If all matter were minimally coupled to $\tilde{g}_{\mu \nu}$, then the equivalence principle would still hold [apart from the current-current finite-range interaction entailed by the direct coupling (5.2)]. However, use of the full connection (5.4) (required for fermions) or allowing matter couplings other than just to $\tilde{g}_{\mu \nu}$ generically violates the equivalence principle. A convenient parametrization of equivalence violation is to allow different types of matter to couple to different "metrics" $\tilde{g}_{\mu \nu}^{A}$ and affinities $\hat{\Gamma}_{\mu \nu}^{A \lambda}$ labeled by different parameters $\left(a_{A}, b_{A}\right)$ in (5.3) and $\left(c_{A}, d_{A}\right)$ in (5.4).

A stronger, dilaton-inspired way of violating the equivalence principle would be to have the material Lagrangians multiplied by factors of the form $(1+$ $e B^{\alpha \beta} B_{\alpha \beta}$ ). Finally, recent studies in NGT [24-26] also 
suggest the possibility of violating the equivalence principle by adding nonmetric couplings between $B_{\mu \nu}$ and the electromagnetic field, say

$$
\mathcal{L}_{B F}^{\prime}=\alpha\left(B_{\mu \nu} F^{\mu \nu}\right)^{2} \text {. }
$$

[The other possible quadratic couplings $B^{\mu \alpha} B_{\alpha}^{\nu} F_{\mu \beta} F_{\nu}{ }^{\beta}$ and $B^{\alpha \beta} B_{\alpha \beta} F_{\mu \nu} F^{\mu \nu}$ are already covered by the parameters $a$ and $e$ introduced above, and the terms quadratic in the dual $F_{\mu \nu}^{*}$, e.g., $\left(B_{\mu \nu} F^{* \mu \nu}\right)^{2}$, are equivalent to these; a linear mixing $B_{\mu \nu} F^{\mu \nu}$ would lead to unacceptable asymptotic behavior.] Note that even if there is only one metric $\tilde{g}_{\mu \nu}$ which couples universally to matter, then (5.5) violates not only equivalence but also local Lorentz invariance and local isotropy of space. Indeed, by Eq. (5.2), fermionic currents outside a local laboratory will generate an external, macroscopic, $B_{\mu \nu}$ field that will introduce, via (5.5), preferred spacetime directions in all physical effects involving electromagnetism.

We propose, then, a general multiparameter gravity model defined by a Lagrangian of the type

$$
\begin{aligned}
\mathcal{L}= & \mathcal{L}_{G}+\mathcal{L}_{B}+\mathcal{L}_{J} \\
& +\sum_{A}\left(1+e_{A} B^{2}\right) \mathcal{L}_{A}\left[\psi_{A}, \tilde{g}_{\mu \nu}^{A}, \hat{\Gamma}_{\mu \nu}^{A \lambda}\right]+\mathcal{L}_{B F}^{\prime}
\end{aligned}
$$

where

$$
\mathcal{L}_{G}=\frac{\sqrt{-G}}{4 \kappa^{2}} \bar{R}(G)
$$

is the Einstein Lagrangian $\left(\kappa^{2} \equiv 4 \pi G_{\text {Newton }}\right)$,

$$
\mathcal{L}_{B}=-\frac{\sqrt{-G}}{\kappa^{2}}\left[\frac{1}{12} H^{2}+\frac{1}{4} \mu^{2} B^{2}\right]
$$

is the massive ghost-free $B$ Lagrangian (with $B$ dimensionless in these gravitational units), and $\mathcal{L}_{A}$ denotes the Lagrangian describing some matter field $\psi_{A}$ minimally coupled to a metric and a connection, e.g.,

$$
\mathcal{L}_{\text {Dirac }}=\sqrt{-G} \bar{\psi} \gamma^{a}\left(\tilde{e}_{a}^{\mu} \partial_{\mu}+\frac{1}{2} \hat{\Gamma}_{c a}^{b} \sigma_{b}^{c}\right) \psi
$$

Here $\tilde{e}_{a}^{\mu}$ is the vierbein and $\hat{\Gamma}_{a c}^{b}$ a general connection (5.4); latin letters denote local indices. The other terms in (5.6) denote the coupling terms already introduced, (5.2) and (5.5). The only dimensionful "gravitational" parameters entering the general class of Lagrangians (5.6) are the bare gravitational coupling constant $\kappa$ and the mass $\mu$ of $B$ (with dimensions of inverse length in ordinary units). The dimensionless parameters are the (possibly matterdependent) numbers $\left(a_{A}, \ldots, e_{A}\right), \alpha$, together with the current coupling constant $f$ and various mixing angles defining the given current. Such angles have been discussed in the "fifth force" literature, where it was noted that the current $J^{\mu}$ could contain several free weight parameters (e.g., distinguishing the lepton numbers belonging to different families). In the case of coupling to ordinary macroscopic matter, it is enough to introduce one mixing angle [27], say $\theta_{5}$, such that the total "charge" is

$$
q_{5} \equiv \int \sqrt{-G} d^{3} x J^{0}=B \cos \left(\theta_{5}\right)+L \sin \left(\theta_{5}\right)
$$

where $B=N+Z$ is the baryon number and $L=Z$ the total lepton number. (When exploring cosmological aspects of the present class of theories, one also has the possibility of introducing a different coupling to fermionic dark matter.)

We certainly do not claim that such a general model recommends itself by its elegance, but we propose it as a replacement for NGT to provide a foil for general relativity, useful for suggesting some intriguing possibilities in experimental gravitation. In addition, our general approach, motivated as it is by string theory, nonsymmetric geometric models and Einstein-Cartan-type theories, is likely to encompass more possibilities.

In order to outline the main types of observational consequences of our general model, let us study the $B$-field equations. If $K_{\mu \nu}$ denotes the effective source for $B_{\mu \nu}$ (including nonlinearities), i.e.,

$K^{\mu \nu}=\frac{2}{\sqrt{-G}} \frac{\delta}{\delta B_{\mu \nu}}\left[\mathcal{L}_{J}+\sum_{A}\left(1+e_{A} B^{2}\right) \mathcal{L}_{A}+\mathcal{L}_{B F}^{\prime}\right]$

we can formally write the $B$-field equations as

$$
-\bar{\nabla}^{\lambda} H_{\lambda \mu \nu}+\mu^{2} B_{\mu \nu}=\kappa^{2} K_{\mu \nu} .
$$

The identity $\bar{\nabla}^{\lambda} \bar{\nabla}^{\mu} H_{\lambda \mu \nu} \equiv 0$ implies

$$
\bar{\nabla}^{\nu} B_{\mu \nu}=\frac{\kappa^{2}}{\mu^{2}} \bar{\nabla}^{\nu} K_{\mu \nu}
$$

and hence we obtain the following inhomogeneous KleinGordon equation ${ }^{8}$ for $B_{\mu \nu}$

$$
(\bar{\Delta} B)_{\mu \nu}+\mu^{2} B_{\mu \nu}=\kappa^{2}\left(K_{\mu \nu}+\frac{1}{\mu^{2}} \partial_{[\mu} \bar{\nabla}^{\alpha} K_{\nu] \alpha}\right)
$$

where $\bar{\Delta}$ denotes the Hodge-de Rham Laplacian in the metric $G_{\mu \nu}$, differing from the ordinary Laplacian by curvature terms:

$$
(\bar{\Delta} B)_{\mu \nu} \equiv-G^{\alpha \beta} \bar{\nabla}_{\alpha} \bar{\nabla}_{\beta} B_{\mu \nu}+(\bar{R} B)_{\mu \nu} .
$$

If $K_{\mu \nu}$ were a linear ( $B$-independent) source, the interaction mediated by $B_{\mu \nu}$ would be equivalent to that due to a massive vectorial (Proca) field. This equivalence is most easily seen in first-order formalism. Indeed, let us start from the first-order form of the massive $B$ theory with both potential and field-strength (Pauli-type) coupling to external sources,

\footnotetext{
${ }^{8}$ Let us note in passing the singularity of the massless limit as made evident by the $1 / \mu^{2}$ factors in the right-hand sides of (5.13) and (5.14). However, this singular behavior concerns mainly nonlinear $O\left(\kappa^{4}\right)$ terms in $B$ because, as is clear from (5.2), the leading source term, $\sqrt{-G} K^{\mu \nu}=f \partial_{\lambda} J^{* \lambda \mu \nu}+$ $O\left(\kappa^{2} f\right)$, is conserved: $\bar{\nabla}_{\nu} K^{\mu \nu}=0+O\left(\kappa^{2} f\right)$.
} 


$$
\begin{aligned}
\mathcal{L}(B, H)=- & \frac{\sqrt{-G}}{\kappa^{2}}\left[-\frac{1}{12} H^{2}+\frac{1}{4} \mu^{2} B^{2}\right. \\
& \left.+\frac{1}{6} H^{\lambda \mu \nu} \partial_{[\lambda} B_{\mu \nu]}\right] \\
& +\frac{1}{2} \sqrt{-G} B_{\mu \nu} K^{\mu \nu}+\frac{1}{6} \sqrt{-G} H_{\lambda \mu \nu} L^{\lambda \mu \nu} .
\end{aligned}
$$

Defining $^{9} \quad F_{\mu \nu}=\sqrt{-G} \quad \frac{\mu}{2 \kappa} \epsilon_{\mu \nu \alpha \beta} B^{\alpha \beta}, \quad A_{\mu}=$ $-\frac{\sqrt{-G}}{6 \mu \kappa} \epsilon_{\mu \alpha \beta \gamma} H^{\alpha \beta \gamma}$, one obtains

$$
\begin{aligned}
\mathcal{L}(A, F)= & \sqrt{-G}\left[\frac{1}{4} F^{2}-\frac{1}{2} \mu^{2} A^{2}-\frac{1}{2} F^{\mu \nu} \partial_{[\mu} A_{\nu]}\right] \\
& +\sqrt{-G} A_{\mu} J_{\text {Proca }}^{\mu}+\frac{1}{2} \sqrt{-G} F_{\mu \nu} M_{\text {Proca }}^{\mu \nu}
\end{aligned}
$$

which is the first-order form of a Proca field coupled to

$$
J_{\text {Proca }}^{\mu}=-\frac{\kappa \mu}{6} \frac{\epsilon^{\mu \alpha \beta \gamma}}{\sqrt{-G}} L_{\alpha \beta \gamma}, \quad M_{\text {Proca }}^{\mu \nu}=\frac{\kappa}{2 \mu} \frac{\epsilon^{\mu \nu \alpha \beta}}{\sqrt{-G}} K_{\alpha \beta} .
$$

Equation (5.14) can formally be solved by successive iterations in powers of $\kappa^{2}$,

$$
\begin{gathered}
B_{\mu \nu}=\kappa^{2} B_{\mu \nu}^{(1)}+\kappa^{4} B_{\mu \nu}^{(2)}+\cdots \\
G_{\mu \nu}=G_{\mu \nu}^{(0)}+\kappa^{2} G_{\mu \nu}^{(1)}+\cdots
\end{gathered}
$$

where, for simplicity, we shall consider a flat background metric, $G_{\mu \nu}^{(0)}=\eta_{\mu \nu}$. Inserting the $O\left(\kappa^{0}\right)$ source read off from (5.2),

$$
K_{\mu \nu}=-f \epsilon_{\mu \nu \alpha \beta} \partial^{\alpha} J^{\beta}+O\left(\kappa^{2} f\right)
$$

one gets

$B_{\mu \nu}=\kappa^{2} f\left(\square-\mu^{2}\right)^{-1}\left[\epsilon_{\mu \nu \alpha \beta} \partial^{\alpha} J^{\beta}\right]+O\left(\kappa^{4}\right)$.

Moreover, our previous results (5.15)-(5.17) [remembering the Pauli form (5.2) of the lowest-order matter coupling] show that the lowest order matter-matter coupling mediated by the $B$ field (5.21) is equivalent (apart from contact terms) to a vectorial "fifth-force" interaction coupled to the fermion current $J^{\mu}$ [with total charge (5.10)] with dimensionless coupling constant

$$
g_{5}=\kappa \mu f .
$$

Note the proportionality of the strength of the effective fifth-force coupling to the inverse range of the interaction. On the other hand, recent experimental work on possible fifth forces has put bounds on $g_{5}$ that, though becoming more stringent as the range $\mu^{-1}$ increases, stay finite as

\footnotetext{
${ }^{9}$ Note again the singularity of the massless limit, which we know turns the $B$ field into a massless scalar.
}

$\mu$ vanishes. To quote some numbers, the dimensionless quantity $\alpha_{5}=g_{5}^{2} /\left(\kappa^{2} m_{N}^{2}\right)$ (where $m_{N}$ denotes, say, one atomic mass unit) was found to be bounded by $\lesssim 10^{-3}$ when $\mu^{-1}=1 \mathrm{~m}, \lesssim 10^{-5}$ when $\mu^{-1}=1 \mathrm{~km}$, and $\lesssim 10^{-8}$ for $\mu^{-1}>10^{4} \mathrm{~km}$ [assuming a coupling to baryon number, i.e., $\theta_{5}=0$ in (5.2); see [28] for precise numbers, the cependence on the mixing angle, and further references]. The important points for our present phenomenological purpose are that the strength $f$ of our basic coupling is unbounded as the range increases, and that the magnitude (5.21) of the $B$ field itself is primarily proportional to $f$ and independent of the range $\mu^{-1}$ (until the nonlinear terms $\propto \kappa^{2} \mu^{-2}$ come into play). This therefore opens the possibility of having a $B$ field of "gravitational" strength, contributing significant new macroscopic forces, while still keeping compatibility with the existing stringent bounds on possible composition-dependent effects in Newtonian gravity. ${ }^{10}$

To discuss the leading observational consequences of our general framework, it is convenient to replace the fundamental dimensionless coupling constant $f$ by the auxiliary quantity

$$
\lambda \equiv \frac{f}{m_{N}} \approx(2 f) \times 10^{-14} \mathrm{~cm}
$$

which has the dimension of length and which couples $B$ to $m_{N} J^{\mu}$, i.e., roughly speaking, to the baryon restmass density. Then we introduce, as leading "short-range gravitational potential," the quantity

$$
V \equiv \frac{\kappa^{2} m_{N} J^{0}}{\mu^{2}-\square}
$$

(which is approximately equal to the Newtonian potential $U \equiv-\kappa^{2} \Delta^{-1} T^{00}$ when the range is large). In units where only the velocity of light is set equal to one, the potential $V$ is, like $U$, dimensionless. Then the leading components of $B_{\mu \nu}$ (for slowly moving sources $J^{i} / J^{0} \sim v \ll 1$ ) are the "magnetic" ones,

$$
B_{0 i}^{*}=\frac{1}{2} \epsilon_{i j k} B_{j k}=\lambda \partial_{i} V
$$

the electric components $B_{0 i}=\frac{1}{2} \epsilon_{i j k} B_{j k}^{*}$ being $v$ times smaller.

We can reexpress the auxiliary coupling length $\lambda$ in terms of experimentally constrained fifth-force quantities as

$$
\lambda=\sqrt{\alpha_{5}} \mu^{-1}=\sqrt{\alpha_{5}} \times(\text { range of } B) .
$$

This shows that $\lambda$ can take any macroscopic value, as long as the range of $B$ is large enough (the corresponding value of $f$ is then large, e.g., $f \sim 10^{19} \sim m_{P} / m_{N}$ for $\lambda \sim 1$

\footnotetext{
${ }^{10}$ Note that, in view of our above proof of equivalence of linear $B$ couplings to vectorial ones, this possibility exists only if there are explicit $B$-dependent terms in the $\mathcal{L}_{A}$-type Lagrangians (i.e., some parameters among $a_{A}, \ldots, e_{A}, \alpha$ must be nonzero).
} 
$\mathrm{km}$, where $m_{P}$ denotes the Planck mass).

At order $\kappa^{4}$, the $B$ field has two kinds of metricgravitational effects. On the one hand the $\mathcal{L}_{B}$-part of the action contributes $O\left(B^{2}\right)$ source terms in the Einstein equations for $G_{\mu \nu}$, and on the other hand the $B^{2}$ terms in the definition (5.3) contribute directly to the physical metric in which each type of matter "falls." Both types of terms will contribute terms of the form

$$
-\frac{1}{2} \ln \left(-\tilde{g}_{00}\right)=U+k\left(\lambda \partial_{i} V\right)^{2}+\cdots,
$$

for some numerical constant $k$, to the logarithm of the time-time component of the metric (which plays the role of a "quasi-Newtonian" potential). (We have omitted $\mu$ dependent terms on the right that we do not attempt to discuss here.) Hence, we see that, when $\mu^{-1}$ is greater than the characteristic distances of the problem under consideration, there will be a "van der Waals" higherpower-type contribution $\sim \kappa^{4} \lambda^{2} M^{2} / r^{4}$ to the effective gravitational potential (this arises separately from the $\sim$ $\kappa^{2} \lambda^{2} \mu^{2} M / r$ composition-dependent fifth force potential).

If we consider the minimal models with a universal metric coupling $\left(a_{A}=a, b_{A}=b\right.$ for all $A$ 's, $c_{A}=d_{A}=$ $e_{A}=\alpha=0$ ) the main new effects carried by the $B$ field (apart from the fifth-force one) will be associated with the $(\lambda \nabla V)^{2}$ potential of (5.27) (as stated above, this requires nonzero explicit $B^{2}$ terms in the physical metric; $a^{2}+b^{2} \neq 0$ ). As $\lambda$ is a universal length, these effects should be strongest in the smallest and most strongly self-gravitating $(V \lesssim U \sim 1)$ objects, namely neutron stars. Such models could therefore offer interesting foils for the strong-field regime of general relativity. A detailed study is needed to see if they could be tested by means of binary pulsar data, following the methodology of [29]. If $\lambda$ is large enough these terms could be tested in the solar system as $(\lambda / r)^{2}$ fractional deviations of the post-Newtonian effects. In this, and the following, discussion we assume a range larger than the characteristic distances of the system considered. We are aware that this might be difficult to achieve beyond certain limits because of the formal $\mu^{-2}$ singularity apparent in (5.12), (5.13). A closer study of what happens when $\mu$ becomes very small is needed.

If we turn now to the most general models of the type (5.6) they will predict, beyond the effects already discussed, the following: (i) Violations of the weak equivalence principle, when the $a_{A}$ 's and $b_{A}$ 's are not the same for all material fields or interactions, or when $e_{A} \neq 0$. These violations will be proportional to the $(\lambda \nabla V)^{2}$ type of terms. (ii) Violations of local Lorentz invariance and existence of peculiar electromagnetic phenomena when $\alpha \neq 0$, as recently pointed out in $[26,30]$. (iii) "Monopoledipole" coupling of the (quantum) spin of elementary particles to the macroscopic $B$ field generated by, say, the baryons in the Earth. The latter coupling is akin to those proposed in [31]. In our model this type of coupling comes from the possible additional torsion terms in (5.4). Indeed, a Dirac particle couples to torsion via

$$
\frac{1}{2} \sqrt{-G} \bar{\psi} \gamma^{\mu} T_{\beta \mu}^{\alpha} \sigma_{\alpha}{ }^{\beta} \psi
$$

One can see that the terms written in (5.4) give an interaction of order $v \lambda \partial^{2} V \sigma$ where $\sigma$ is the spin and $v$ a small velocity. Had one introduced terms $c_{*} \widetilde{\nabla}^{\lambda} B_{\mu \nu}^{*}+$ $d_{*} \tilde{g}^{\lambda \alpha} \partial_{[\alpha} B_{\mu \nu]}^{*}$ on the right-hand side of (5.4), the coupling would have been of the full order $\lambda \partial^{2} V \sigma$ without velocity factors. As was briefly mentioned at the beginning of this section, one could also think of adding nonderivative couplings of $B_{\mu \nu}$ to some microscopic polarization tensor, i.e., $\sim B_{\mu \nu}\left(\bar{\psi} \sigma^{\mu \nu} \psi\right)$. The latter coupling would contribute interactions proportional to $\nabla V \sigma$.

In summary, our new gravity models incorporating a finite-range antisymmetric tensor field are consistent, and they provide a vast reservoir of interesting phenomenological possibilities in experimental gravitation.

\section{ACKNOWLEDGMENTS}

T.D. thanks G.W. Gibbons for fruitful discussions in the early stages of this work. We are grateful to A. Lichnerowicz for an informative conversation on earlier literature, and to C.M. Will for a suggestion. The work of S.D. and J.M. was supported in part by NSF Grant No. PHY88-04561; S.D. thanks IHES for hospitality.

\section{APPENDIX A: THE $B_{\mu \nu}$ EXPANSION}

From the general form of $g_{\mu \nu}$,

$$
g_{\mu \nu}=G_{\mu \nu}+B_{\mu \nu}+\alpha B_{\mu \alpha} B^{\alpha}{ }_{\nu}+\beta B^{2} G_{\mu \nu}+O\left(B^{3}\right),
$$

we easily determine its inverse and determinant,

$$
g^{\mu \nu}=G^{\mu \nu}+B^{\mu \nu}+(1-\alpha) B^{\mu \alpha} B_{\alpha}^{\nu}-\beta B^{2} G^{\mu \nu}+O\left(B^{3}\right),
$$

$$
\sqrt{-g}=\sqrt{-G}\left[1+\frac{1}{2}\left(\frac{1}{2}-\alpha+\beta D\right) B^{2}\right]+O\left(B^{4}\right) .
$$

Here we are using the notation $B^{\mu}{ }_{\nu} \equiv G^{\mu \alpha} B_{\alpha \nu}=-B_{\nu}{ }^{\mu}$ and $B^{2} \equiv B^{\mu \nu} B_{\mu \nu}$. Consider the defining relation for the affinity as a function of the metric,

$$
\partial_{\lambda} g_{\mu \nu}-\Gamma_{\mu \lambda}^{\alpha} g_{\alpha \nu}-\Gamma_{\lambda \nu}^{\alpha} g_{\mu \alpha}=0 \text {. }
$$

On introducing the expansion $\Gamma_{\mu \nu}^{\lambda}=\sum_{n \geq 0}\left(S_{\mu \nu}^{(n) \lambda}+\right.$ $A_{\mu \nu}^{(n) \lambda}$ ), [where the superscript $(n)$ denotes the order in $B_{\mu \nu}$, while $S$ and $A$ denote symmetric and antisymmetric parts, respectively] into (A4), we easily find $A_{\mu \nu}^{(0) \lambda}=0$ and $S_{\mu \nu}^{(0) \lambda}=\{\stackrel{\lambda}{\mu \nu}\}(G)$, the Christoffel symbol with respect to the "background" $G_{\mu \nu}$. Matching higher orders, we have $S_{\mu \nu}^{(1) \lambda}=0$ and

$$
A_{\mu \nu}^{(1) \lambda}=\frac{1}{2}\left(\bar{\nabla}^{\lambda} B_{\mu \nu}-\bar{\nabla}_{\mu} B_{\nu}{ }^{\lambda}-\bar{\nabla}_{\nu} B_{\mu}^{\lambda}\right)
$$

where, as always, the bar denotes operations with respect to the background $G_{\mu \nu}$. The higher powers are defined by 


$$
\begin{aligned}
S_{\mu \lambda}^{(2 n) \alpha} G_{\alpha \nu}+S_{\lambda \nu}^{(2 n) \alpha} G_{\mu \alpha}= & -A_{\mu \lambda}^{(2 n-1) \alpha} B_{\alpha \nu} \\
& -A_{\lambda \nu}^{(2 n-1) \alpha} B_{\mu \alpha} \\
A_{\mu \lambda}^{(2 n+1) \alpha} G_{\alpha \nu}+A_{\lambda \nu}^{(2 n+1) \alpha} G_{\mu \alpha}= & -S_{\mu \lambda}^{(2 n) \alpha} B_{\alpha \nu} \\
& -S_{\lambda \nu}^{(2 n) \alpha} B_{\mu \alpha}
\end{aligned}
$$

For simplicity we have written the equations (A6) as if $g_{\mu \nu}^{(n)}=0, n \geq 2$ in (2.5); the modification for nonzero field redefinition is trivially accomplished, and does not alter (A5). Clearly (A6) may be solved iteratively, to obtain the expansion of $\Gamma_{\mu \nu}^{\lambda}$ in powers of $B_{\mu \nu}$.

The more general equation (2.15) for the affinity leads to a similar expansion. Using it, we obtain to lowest order

$$
\left(1+\frac{a+b+c+d}{2}\right) A_{\mu[\lambda}^{(0) \alpha} G_{\nu] \alpha}=0 .
$$

Thus generically we find $A_{\mu \nu}^{(0) \lambda}=0$ and, going on as before, obtain an iterative solution. The exceptional case where the affinity cannot be determined is exactly that listed in the text, $a+b+c+d=-2$.

With the above results we may easily write the expansion of the geometric quantities defined in the text. In particular, we find

$$
\begin{aligned}
R_{\mu \nu}(g)= & \bar{R}_{\mu \nu}(G)+\frac{1}{2} \bar{\nabla}^{\alpha} \bar{\nabla}_{\alpha} B_{\mu \nu}-\frac{1}{2} \bar{\nabla}^{\alpha} \bar{\nabla}_{[\mu} B_{\nu] \alpha} \\
& -\bar{\nabla}_{\nu} \bar{\nabla}^{\alpha} B_{\mu \alpha}+O\left(B^{2}\right) \\
T_{\mu \nu}^{\lambda}= & A_{\mu \nu}^{(1) \lambda}+O\left(B^{3}\right) \\
\Gamma_{\mu}= & \bar{\nabla}^{\alpha} B_{\mu \alpha}+O\left(B^{3}\right), \\
\sqrt{-g} g^{\mu \nu} R_{\mu \nu}(g)= & \sqrt{-G} \bar{R}(G)-\frac{1}{12} \sqrt{-G} H^{\mu \nu \lambda} H_{\mu \nu \lambda} \\
& +\frac{1}{2}\left[\frac{1}{2}-\alpha+(D-2) \beta\right] \sqrt{-G} \bar{R}(G) B^{2} \\
& -\alpha \sqrt{-G} \bar{R}_{\mu \nu}(G) B^{\mu \alpha} B_{\alpha}^{\nu} \\
& -\sqrt{-G} \bar{R}_{\mu \nu \alpha \beta} B^{\mu \alpha} B^{\nu \beta} \\
& +\operatorname{total~derivative+} O\left(B^{4}\right) \\
\sqrt{-g} g^{\mu \nu} \Gamma_{\mu} \Gamma_{\nu}= & \sqrt{-G} G^{\mu \nu} \bar{\nabla}^{\alpha} B_{\mu \alpha} \bar{\nabla}^{\beta} B_{\nu \beta}+O\left(B^{4}\right), \\
\sqrt{-g} g^{\mu \nu} T_{\mu \lambda}^{\alpha} T_{\alpha \nu}^{\lambda}= & -\frac{1}{12} \sqrt{-G} \bar{H}^{\mu \nu \lambda} \bar{H}_{\mu \nu \lambda} \\
& -\sqrt{-G} G_{\mu \nu} \bar{\nabla}_{\beta} B^{\mu \alpha} \bar{\nabla}_{\alpha} B^{\nu \beta}+O\left(B^{4}\right) .
\end{aligned}
$$

We also use implicitly the fact, which follows from the " $g^{\mu \nu}$ trace" of (A4), that

$$
\Gamma_{(\lambda \alpha)}^{\alpha}(g)=\partial_{\lambda} \ln (-g)
$$

and so

$$
P_{\mu \nu}(\Gamma(g))=-\partial_{[\mu} \Gamma_{\nu]}(g) .
$$

It is then easy to show that in second order, up to a total derivative,

$$
\sqrt{-g} g^{\mu \nu} P_{\mu \nu}=-2 \sqrt{-g} g^{\mu \nu} \Gamma_{\mu} \Gamma_{\nu}
$$

\section{APPENDIX B: FIRST- AND SECOND-ORDER FORMS OF NGT}

The first-order NGT action may be taken as

$$
\mathcal{L}^{(1)}=\sqrt{-g} g^{\mu \nu} R_{\mu \nu}(\Gamma) \text {. }
$$

We saw in Sec. II that its equations of motion are equivalent to those of the second-order action

$$
\mathcal{L}^{(2)}=\sqrt{-g} g^{\mu \nu} R_{\mu \nu}(g)-b_{\nu} \partial_{\mu}\left(\sqrt{-g} g^{[\mu \nu]}\right) .
$$

The difficulties of this model at quadratic order and beyond are chronicled in detail in Sec. IV. Our aim here is rather to clearly demonstrate the equivalence of the above two formulations, since doubts have been raised on this question [7]. It will suffice to do so for the linearized theory about a flat background since this exhibits the kinematics of degrees of freedom which is at issue. We begin with the second-order action, which we expand in powers of $g_{\mu \nu}-\eta_{\mu \nu}=s_{\mu \nu}+B_{\mu \nu}$. Using Appendix A we find

$$
\mathcal{L}=\mathcal{L}_{E}^{(2)}(s)+\mathcal{L}_{B}^{(2)}(B)+O\left((B, s)^{3}\right),
$$

where $\mathcal{L}_{E}$ is the linearized Einstein action and

$$
\mathcal{L}_{B}^{(2)}=-\frac{1}{12} H_{\mu \nu \lambda}^{2}-2 b_{\nu} \partial_{\mu} B^{\mu \nu} .
$$

This may be recognized as a gauge fixed form of the $H^{2}$ action akin to the Nakanishi-Lautrup [32] gauge fixing in electrodynamics; its degrees of freedom are well understood. For completeness, we summarize the analysis of the equations of motion from (B4), which are equivalent to

$$
\begin{aligned}
\square B_{\mu \nu}+2 \partial_{[\mu} b_{\nu]} & =0 \\
\partial^{\alpha} B_{\mu \alpha} & =0 .
\end{aligned}
$$

The divergence of (B5) states that $b_{\mu}$ obeys Maxwell's equations

$$
\partial^{\mu} \partial_{[\mu} b_{\nu]}=0 \text {. }
$$

Suppose now that we are given the $2 \times(D-2)$ Cauchy data [corresponding to $(D-2)$ dynamical degrees of freedom] required to specify (modulo its gauge invariance) $b_{\mu}$ from (B7); then (B5) implies

$$
\square \partial^{\mu} B_{\mu \nu}=0 \text {, }
$$

and so the constraint (B6) can be incorporated as a set of relations among the Cauchy data of (B5). That is, requiring $\partial^{\alpha} B_{\mu \alpha}$ and its first time derivative to vanish at $t=0$ ensures that it obeys (B6) for all time. In turn, these relations determine the Cauchy data for $B_{0 i}$ according to

$$
\begin{aligned}
& \left.\left(\partial_{0} B_{0 i}=\partial^{j} B_{j i}\right)\right|_{t=0}, \\
& \left.\left(\Delta B_{i 0}=\partial^{j} \partial_{0} B_{i j}-2 \partial_{i} b_{0}+2 \partial_{0} b_{i}\right)\right|_{t=0},
\end{aligned}
$$

(from which $\partial^{i} B_{0 i}=0$ follows). Hence the independent Cauchy data are the $2(D-2)$ for $b_{\mu}$ together with the 
$(D-1)(D-2)\left\{B_{i j}, \dot{B}_{i j}\right\}$. Thus the total number of degrees of freedom is $\frac{1}{2}(D+1)(D-2)$, namely 5 in $D=4$. (This is in agreement with an early estimate by Einstein, in the third reference of [2].) The apparent paradoxthat this cannot be the correct count for a gauge-fixed theory known to have but one degree of freedom in $D=$ 4 -is cleared up by the following remarks. First, in the gauge fixing, $b_{\mu}$ may be eliminated initially (and hence consistently for all time). The other is that (B6) has a residual gauge invariance under $\delta B_{\mu \nu}=\partial_{[\mu} \epsilon_{\nu]}$, for $\epsilon_{\mu}$ obeying Maxwell's equations. Hence, as usual, the two longitudinal modes simply decouple, leaving the single scalar degree of freedom.

The above analysis agrees completely with that of [33], which has however been criticized as deviating from NGT by its use of second-order form. Let us therefore explicitly construct the canonical formulation directly from the first-order one, so that the degrees of freedom may be counted directly. Expanding (B1) to quadratic order in the fields we have $\left(\sqrt{-g} g^{\mu \nu} \equiv \eta^{\mu \nu}+h^{\mu \nu}+B^{\mu \nu}\right)$,

$$
\mathcal{L}_{2}^{(1)}=\left(h^{\mu \nu}+B^{\mu \nu}\right) \partial_{[\lambda} \Gamma_{\mu \nu]}^{\lambda}-\eta^{\mu \nu} \Gamma_{\mu[\lambda}^{\alpha} \Gamma_{\alpha \nu]}^{\lambda},
$$

where only $[\lambda \nu]$ are antisymmetrized. Decomposing $\Gamma_{\mu \nu}^{\lambda}$ into its symmetric $(S)$ and antisymmetric $(A)$, parts, we obtain

$$
\begin{aligned}
\mathcal{L}_{2}^{(1)}= & h^{\mu \nu} \partial_{[\lambda} S_{\nu] \mu}^{\lambda}-\eta^{\mu \nu} S_{\mu[\lambda}^{\alpha} S_{\nu] \alpha}^{\lambda}-B^{\mu \nu} \partial_{[\lambda} A_{\nu] \mu}^{\lambda} \\
& +\eta^{\mu \nu} A_{\mu[\lambda}^{\alpha} A_{\nu] \alpha}^{\lambda}-h^{\mu \nu} \partial_{\nu} A_{\mu \lambda}^{\lambda} \\
& -B^{\mu \nu} \partial_{\nu} S_{\mu \lambda}^{\lambda}+\eta^{\mu \nu} S_{\mu \nu}^{\alpha} A_{\alpha \lambda}^{\lambda} .
\end{aligned}
$$

Only the last three terms in (B11) couple the symmetric and antisymmetric components, and do so only through traces of the affinity; hence we may diagonalize just by shifting to $\widetilde{\Gamma}_{\mu \nu}^{\lambda}=\Gamma_{\mu \nu}^{\lambda}+\frac{2}{D-1} \delta_{\mu}^{\lambda} \Gamma_{\nu}$, to make $\widetilde{A}_{\mu \lambda}^{\lambda}=0$.
Since $R_{\mu \nu}(\Gamma)=R_{\mu \nu}(\widetilde{\Gamma})-\frac{2}{D-1} \partial_{[\mu} \Gamma_{\nu]}$, where $\Gamma_{\nu} \equiv A_{\nu \lambda}^{\lambda}$, we get simply $\mathcal{L}_{2}^{(1)}=\mathcal{L}_{E}^{(1)}+\mathcal{L}_{B}^{(1)}$ where $\mathcal{L}_{E}^{(1)}$ is linearized Einstein gravity in first order form (known of course to be equivalent to its second-order one) and

$\mathcal{L}_{B}^{(1)}=B^{\mu \nu} \partial_{\lambda} \widetilde{A}_{\mu \nu}^{\lambda}+\eta^{\mu \nu} \widetilde{A}_{\mu \lambda}^{\alpha} \widetilde{A}_{\nu \alpha}^{\lambda}-\tilde{b}_{\nu} \partial_{\mu} B^{\mu \nu}$

in terms of $\tilde{b}_{\mu} \equiv-\frac{4}{D-1} \Gamma_{\mu}+S_{\mu \lambda}^{\lambda}$.

The canonical analysis of (B12) is completely straightforward, and we find

$$
\begin{aligned}
\mathcal{L}_{B}^{(1)}= & \pi^{i j} \partial_{0} B_{i j}+\pi^{i} \partial_{0} B_{i 0}-B^{i j} \partial_{i} \pi_{j}+\tilde{b}_{0} \partial^{i} B_{i 0} \\
& +\widetilde{A}_{0 i}^{j}\left[2 \partial_{j} B_{0 i}+2 \pi_{i j}\right]-\widetilde{A}_{0 i}^{j} \widetilde{A}_{0 j}^{i} \\
& +\widetilde{A}_{0 i}^{0}\left[\left(-\frac{2}{D-2}-2\right) \partial_{j} B^{i j}\right]+\frac{D-1}{D-2}\left(\widetilde{A}_{0 i}^{0}\right)^{2} \\
& +\bar{A}_{i j}^{k}\left[-\partial_{k} B^{i j}\right]-\bar{A}_{i j}^{k} \bar{A}_{k i}^{j} .
\end{aligned}
$$

Here the conjugate momenta have been identified via

$$
\pi_{i j}=-\widetilde{A}_{i j}^{0}, \quad \pi_{i}=-\tilde{b}_{i}-2 \widetilde{A}_{0 i}^{0},
$$

we have separated out the trace pieces

$$
\widetilde{A}_{i j}^{k}=\bar{A}_{i j}^{k}+\frac{1}{D-2} \delta_{[j}^{k} A_{i]}, \quad A_{i}=\widetilde{A}_{i j}^{j},
$$

and the $\widetilde{A}_{\mu \lambda}^{\lambda}=0$ constraint has been applied. The " $p \dot{q}$ " terms in (B13) show that $\left(\widetilde{A}_{0 i}^{j}, \widetilde{A}_{0 i}^{0}, \bar{A}_{i j}^{k}\right)$ are all auxiliary fields, while the canonical pairs $\left(\pi^{i j}, B_{i j}\right)$, $\left(\pi^{i}, B_{0 i}\right)$ obey the one constraint $\partial^{i} B_{0 i}=0$, enforced by the Lagrange multiplier $\tilde{b}_{0}$. This means there are $\frac{1}{2}(D-1)(D-2)+(D-1)-1=\frac{1}{2}(D+1)(D-2)$ degrees of freedom, all completely equivalent to the second-order analysis.
[1] A. Einstein, Sitz. Preuss. Akad. Wiss., 414 (1925).

[2] A. Einstein and E.G. Straus, Ann. Math. 47, 731 (1946); A. Einstein and B. Kaufman, ibid. 62, 128 (1955); A. Einstein, The Meaning of Relativity, 5th ed. (Princeton University Press, Princeton, NJ, 1955).

[3] E. Schrödinger, Proc. R. Ir. Acad. 51A, 163 (1947); Space-Time Structure (Cambridge University Press, Cambridge, England, 1950).

[4] A. Lichnerowicz, Théories Relativistes de la Gravitation et de l' Electromagnétisme (Masson, Paris, 1955).

[5] M.A. Tonnelat, La Théorie du Champ Unifié d'Einstein (Gauthier-Villars, Paris, 1955).

[6] F. Maurer-Tison, Ann. Sci. Ec. Norm. Suppl. 76, 185 (1959); J. Vaillant, C.R. Acad. Sci. Paris 253, 231 (1961); 253, 1909 (1961).

[7] J. Moffat, Phys. Rev. D 19, 3554 (1979); and in Gravitation 1990: A Banff Summer School, edited by R.B. Mann and P. Wesson (World Scientific, Singapore, 1991).

[8] H. Weyl, Naturwissenschaften 38, 73 (1951).

[9] W. Pauli, Theory of Relativity (Pergamon Press, Oxford, 1958), supplementary note no. 23.

[10] I.V. Ogievetski and V.I. Polubarinov, Yad. Fiz. 4, 231
(1966) [Sov. J. Nucl. Phys. 4, 156 (1967)]; S. Deser, Phys. Rev. 187, 1931 (1969); K. Hayashi, Phys. Lett. 44B, 497 (1973); M. Kalb and P. Ramond, Phys. Rev. D 9, 2274 (1974); E. Cremmer and J. Scherk, Nucl. Phys. B72, 117 (1974).

[11] T. Damour, S. Deser, and J. McCarthy, Phys. Rev. D 45, R3289 (1992).

[12] P.F. Kelly and R.B. Mann, Class. Quantum Grav. 3, 705 (1986); R.B. Mann, Can. J. Phys. 64, 589 (1986); see also R.B. Mann, Class. Quantum Grav. 6, 41 (1989).

[13] N.J. Cornish and J.W. Moffat, University of Toronto Report No. UTPT-91-33, 1991 (unpublished).

[14] R.A. Isaacson, Phys. Rev. 166, 1263 (1968); 166, 1272 (1968).

[15] T. Damour and B. Schmidt, J. Math. Phys. 31, 2441 (1990).

[16] R.M. Wald, Phys. Rev. D 33, 3613 (1986).

[17] P.F. Kelly, Class. Quantum Grav. 8, 1212 (1991).

[18] P.F. Kelly and R.B. Mann, Class. Quantum Grav. 4, 1593 (1987).

[19] P.F. Kelly, Class. Quantum Grav. 9, 1423(E) (1992).

[20] T.P. Krisher, Phys. Rev. D 32, 329 (1985). 
[21] J.W. Moffat and D.C. Tatarski, University of Toronto Report No. UTPT-92-01, January 1992; revised June 1992 (unpublished).

[22] C. Aragone and S. Deser, Nuovo Cimento 57B, 33 (1980).

[23] F.W. Hehl, J. Nitsch, and P. Von der Heyde, in General Relativity and Gravitation, edited by A. Held (Plenum, New York, 1980), Vol. 1, pp. 329-355.

[24] C.M. Will, Phys. Rev. Lett. 62, 369 (1989).

[25] R.B. Mann, J.H. Palmer, and J.W. Moffat, Phys. Rev. Lett. 62, 2765 (1989).

[26] M.D. Gabriel, M.P. Haugan, R.B. Mann, and J.H. Palmer, Phys. Rev. D 43, 2465 (1991).

[27] A. De Rújula, Phys. Lett B 180, 213 (1986).

[28] E.G. Adelberger et al., Phys. Rev. D 42, 3267 (1990).

[29] T. Damour and J.H. Taylor, Phys. Rev. D 45, 1840 (1992).
[30] M.D. Gabriel, M.P. Haugan, R.B. Mann, and J.H. Palmer, Phys. Rev. D 43, 308 (1991); Phys. Rev. Lett. 67, 2123 (1991); Z. Zhou and M.P. Haugan, Phys. Rev. D 45, 3336 (1992).

[31] J. Leitner and S. Okubo, Phys. Rev. 136, B1542 (1964); N.D. Hari Dass, Phys. Rev. Lett. 36, 393 (1976); J.E. Moody and F. Wilczek, Phys. Rev. D 30, 130 (1984); G.W. Gibbons, in Proceedings of the First William Fairbank Meeting on Relativistic Gravitational Experiments in Space, Rome, 1990, edited by M. Demianski et al. (unpublished).

[32] N. Nakanishi, Prog. Theor. Phys. 35, 1111 (1966); B. Lautrup, Mat. Fys Medd. Dan. Vid. Selsk. 35, 11 (1967).

[33] G. Kunstatter, H.P. Leiva, and P. Savaria, Class. Quantum Grav. 1, 7 (1984). 